Research Article

\title{
Free Vibration Characteristics of Moderately Thick Spherical Shell with General Boundary Conditions Based on Ritz Method
}

\author{
Bing Hu, ${ }^{1,2}$ Cong Gao, ${ }^{3}$ Hang Zhang, ${ }^{3}$ Haichao Li $\mathbb{D},{ }^{3}$ Fuzhen Pang, ${ }^{3}$ and Jicai Lang $\mathbb{D}^{3}$ \\ ${ }^{1}$ State Key Laboratory of Ocean Engineering, Shanghai Jiao Tong University, Shanghai 200240, China \\ ${ }^{2}$ China COSCO Shipping Cooperation Limited, Shanghai 200027, China \\ ${ }^{3}$ College of Shipbuilding Engineering, Harbin Engineering University, Harbin 150001, China \\ Correspondence should be addressed to Haichao Li; lihaichao@hrbeu.edu.cn and Jicai Lang; langjicai@hrbeu.edu.cn
}

Received 17 February 2020; Revised 14 August 2020; Accepted 1 October 2020; Published 29 November 2020

Academic Editor: YuRen Wang

Copyright $\odot 2020$ Bing Hu et al. This is an open access article distributed under the Creative Commons Attribution License, which permits unrestricted use, distribution, and reproduction in any medium, provided the original work is properly cited.

\begin{abstract}
In this paper, the Ritz method is adopted to investigate the vibration characteristics of isotropic moderately thick annular spherical shell with general boundary conditions. The energy expressions of the annular spherical shell were established based on the firstorder shear deformation theory (FSDT). The spring stiffness method is introduced to guarantee continuity and simulate various boundary conditions on the basis of the domain decomposition method. Under the current framework, the displacement admissible function along axial direction and circumferential direction of the shell structure are, respectively, expanded as the unified Jacobi polynomials and Fourier series. The final solutions can be obtained according to the Ritz method. The validity of the proposed method is proved by comparing the results of the same condition with those obtained by the finite element method (FEM) and published literatures. The results show that the current method has fast convergence and delightful accuracy through the comparative study. On this basis, the vibration characteristics of isotropic moderately thick annular spherical shell are further studied by a series of numerical examples.
\end{abstract}

\section{Introduction}

The isotropic moderately thick annular spherical shell structure, as a basic component, has been widely used in many fields of engineering, such as drive shaft, cooling towers, water tanks, pressure vessels, and rotor system. In practical engineering, the dynamical behavior and stability are very important for the structural design because of the structure exposed to various complex environments and bear various dynamic loads. Based on this background, it is very important and necessary to investigate the free vibration characteristics of isotropic moderately thick annular spherical shell under general boundary conditions.

For vibration analysis of thin spherical shell, Bryan [1] developed a new method to investigate the differential equations of motion about a thin spherical shell, in which the Legendre polynomials are utilized to obtain the analytical solutions for the spatial differential equation. Xie et al. [2] proposed a unified approach to study the vibration characteristics of spherical of revolution stiffened by rings with T cross-section. Based on Flugge's thin shell theory, Wang et al. [3] presented the vibration characteristics of coupled doubly curved shell structures which subject to arbitrary boundaries. Pang et al. [4] applied the Rayleigh-Ritz method to analyze the free vibration of doubly curved shells. Li and Yuan [5,6] applied quasi-Green's function method to analyze the free vibration of shallow spherical shell based on the fundamental solution and boundary equation of the problem. By using a hybrid finite element method, Menaa and Lakis [7] presented the free vibration characteristics of the spherical shell by uniting the thin shell theory and classical finite element method. By using the separation of variable method, Zaera et al. [8] obtained the free vibration solutions of closed thin spherical. Polyakov et al. [9] derived the formulation of a closed spherical shell to analyze the free vibrations along the thickness direction of structure. According to von Karman's thin shallow shells theory, Thomas et al. $[10,11]$ derived the 
nonlinear vibration equations of shallow spherical shell under large amplitude displacement.

For vibration analysis related to moderately thick spherical shell structures, Tornabene et al. [12-16] applied the Generalized Differential Quadrature (GDQ) method to investigate the free vibration characteristics of the rotating shell structure based on FSDT. Buchanan and Rich [17] figured out the frequency parameters of thick isotropic spherical shells with simple supports by formulating Lagrangian finite element in spherical coordinates. Based on FSDT, Wang et al. [18-21] carried out a great deal of research studies on spherical shell structures under different boundary conditions, and the analytical solutions were obtained by the Ritz method. Jin et al. [22-25] also conducted a large number of studies to analyze free vibration characteristics of spherical shell structures under general boundary conditions based on the Rayleigh-Ritz method, which each admissible functions is invariantly expanded as a modified Fourier series according to the three-dimensional shell theory of elasticity. Hosseini-Hashemi and Fadaee [26] applied the separation of variables method for free vibration analysis of moderately thick spherical shell according to the first-order shear deformation theory. Zenkour [27] expressed the dynamic responses of anisotropic spherical shells under a uniformly distributed transverse load in the frame work of mixed first-order shear deformation theory. Based on higher order shear deformation theory (HSDT), Fazzolari [28] presented the free vibration characteristics of doubly curved laminated composite spherical shallow shells by using the dynamic stiffness method (DSM). Panda et al. [29-32] investigated the nonlinear free vibration characteristics of laminated composite shallow spherical shell using the variational method, and the analytical model was established on the basis of Green-Lagrange nonlinear kinematics. For space reasons, other papers on rotating cylindrical and spherical shells can be found in references [33-35].

It can be concluded that lots of published literatures focused on the free vibration response of thin spherical shell which subject to general edge restraints, and the free vibration characteristics of moderately thick spherical shell structures have been investigated in some research studies. However, to the authors' knowledge, the aforementioned review for vibration solutions of moderately thick annular spherical shell structure with general boundary conditions based on the FSDT is infrequent. Considering this background, it is necessary to analyze the free vibration characteristics of moderately thick annular spherical shell structure with general boundary conditions. In addition, this paper presents a series of unpublished numerical results of the effects on the boundary conditions, shell segments, and structural parameters, which can be used as basic data to provide comparative data for future researchers in this field.

\section{Theoretical Formulations}

2.1. The Mathematical Model of Moderately Thick Annular Spherical Shell. An isotropic moderately thick annular spherical shell with radius $R$ is considered, as shown in
Figure 1. The deformation displacements of the annular spherical shell with respect to the coordinate system can be, respectively, defined by $u, v$, and $w$ in the $\varphi, \theta$, and $\delta$ directions, and the system $(\varphi, \theta, \delta)$ represent axial, circumferential, and normal directions, accordingly. $C_{s}$ and $h$, respectively, represent the geometry center and thickness of the annular spherical shell. $\varphi_{0}$ and $\varphi_{1}$, respectively, denote the center angle correspond to the top and bottom of annular spherical shell. The formulas of this paper are derived on the basis of the domain decomposition method [36-41], the spherical shell is divided into $H$ segments along axial direction, and the artificial springs are arranged at both ends of each segment; the continuity condition and various boundary conditions can be simulated by assigning the stiffness values of springs.

2.2. Energy Expressions of Moderately Thick Annular Spherical Shell. According to FSDT [42-44], the displacements of $i$ th segment of moderately thick annular spherical shell can be written as follows:

$$
\begin{aligned}
\bar{U}^{i}(\varphi, \theta, \delta, t) & =u^{i}(\varphi, \theta, t)+\delta \psi_{\varphi}^{i}(\varphi, \theta, t), \\
\bar{V}^{i}(\varphi, \theta, \delta, t) & =v^{i}(\varphi, \theta, t)+\delta \psi_{\theta}^{i}(\varphi, \theta, t), \\
\bar{W}^{i}(\varphi, \theta, \delta, t) & =w^{i}(\varphi, \theta, t) .
\end{aligned}
$$

The strains of moderately thick annular spherical shell in this study can be written as follows:

$$
\begin{aligned}
\varepsilon_{\varphi}^{i} & =\varepsilon_{\varphi}^{0, i}+\delta \kappa_{\varphi}^{i}, \\
\varepsilon_{\theta}^{i} & =\varepsilon_{\theta}^{0, i}+\delta \kappa_{\theta}^{i}, \\
\gamma_{\varphi \theta}^{i} & =\gamma_{\varphi \theta}^{0, i}+\delta \kappa_{\varphi \theta}^{i}, \\
\gamma_{\varphi \delta}^{i} & =\gamma_{\varphi \delta}^{0, i}, \\
\gamma_{\theta \delta}^{i} & =\gamma_{\theta \delta}^{0, i},
\end{aligned}
$$

where $\varepsilon_{\varphi}^{i}, \varepsilon_{\theta}^{i}, \gamma_{\varphi \theta}^{i}, \gamma_{\varphi \delta}^{0, i}, \gamma_{\theta \delta}^{0, i}, \kappa_{\varphi}^{i}, \kappa_{\theta}^{i}$, and $\kappa_{\varphi \theta}^{i}$ can, respectively, be expressed as below:

$$
\begin{aligned}
\varepsilon_{\varphi}^{0, i} & =\frac{1}{A} \frac{\partial u^{i}}{\partial \varphi}+\frac{v^{i}}{A B} \frac{\partial A}{\partial \theta}+\frac{w^{i}}{R}, \\
\varepsilon_{\theta}^{0, i} & =\frac{1}{B} \frac{\partial \nu^{i}}{\partial \theta}+\frac{u^{i}}{A B} \frac{\partial B}{\partial \varphi}+\frac{w^{i}}{R}, \\
\gamma_{\varphi \theta}^{0, i} & =\frac{A}{B} \frac{\partial}{\partial \theta}\left(\frac{u^{i}}{A}\right)+\frac{B}{A} \frac{\partial}{\partial \varphi}\left(\frac{v^{i}}{B}\right), \\
\kappa_{\varphi}^{i} & =\frac{1}{A} \frac{\partial \psi_{\varphi}^{i}}{\partial \varphi}+\frac{\psi_{\theta}^{i}}{A B} \frac{\partial A}{\partial \theta}, \\
\kappa_{\theta}^{i} & =\frac{1}{B} \frac{\partial \psi_{\theta}^{i}}{\partial \theta}+\frac{\psi_{\varphi}^{i}}{A B} \frac{\partial B}{\partial \varphi},
\end{aligned}
$$




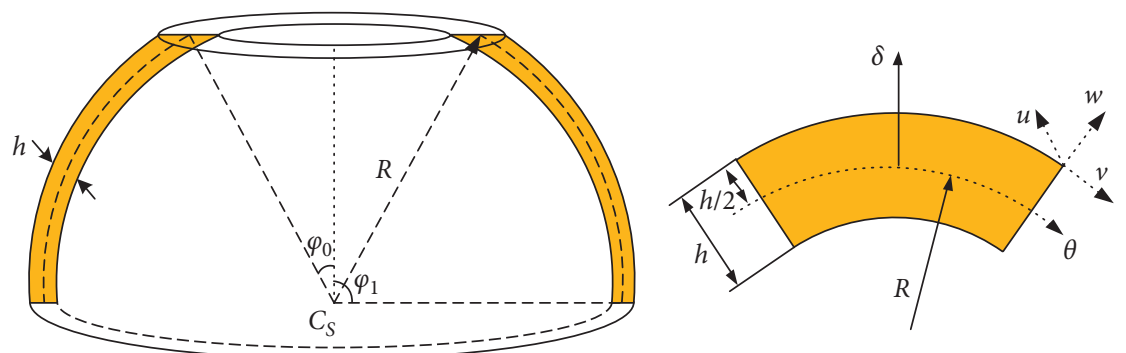

FIGURE 1: Geometry notations and coordinate system of the annular spherical shell.

$$
\begin{aligned}
& \kappa_{\varphi \theta}^{i}=\frac{A}{B} \frac{\partial}{\partial \theta}\left(\frac{\psi_{\varphi}^{i}}{A}\right)+\frac{B}{A} \frac{\partial}{\partial \varphi}\left(\frac{\psi_{\theta}^{i}}{B}\right), \\
& \gamma_{\varphi z}^{0, i}=\frac{1}{A} \frac{\partial w^{i}}{\partial \varphi}-\frac{u^{i}}{R}+\psi_{\varphi}^{i}, \\
& \gamma_{\theta z}^{0, i}=\frac{1}{B} \frac{\partial w^{i}}{\partial \theta}-\frac{v^{i}}{R}+\psi_{\theta}^{i} .
\end{aligned}
$$

In this paper, the symbols $A$ and $B$ are Lamé parameters, and they can be expressed as follows [45, 46]:

$$
\begin{aligned}
& A=R, \\
& B=R \sin \varphi .
\end{aligned}
$$

The stresses of the structure can be written as follows:

$$
\left\{\begin{array}{c}
\sigma_{\varphi}^{i} \\
\sigma_{\theta}^{i} \\
\tau_{\varphi \theta}^{i} \\
\tau_{\varphi \delta}^{i} \\
\tau_{\theta \delta}^{i}
\end{array}\right\}=\left[\begin{array}{ccccc}
Q_{11}(\delta) & Q_{12}(\delta) & 0 & 0 & 0 \\
Q_{12}(\delta) & Q_{22}(\delta) & 0 & 0 & 0 \\
0 & 0 & Q_{66}(\delta) & 0 & 0 \\
0 & 0 & 0 & Q_{66}(\delta) & 0 \\
0 & 0 & 0 & 0 & Q_{66}(\delta)
\end{array}\right]\left\{\begin{array}{c}
\varepsilon_{\varphi}^{i} \\
i \\
\varepsilon_{\theta} \\
\gamma_{\varphi \theta}^{i} \\
\gamma_{\varphi \delta}^{i} \\
\gamma_{\theta \delta}^{i}
\end{array}\right\},
$$

where symbols of $\sigma$ and $\tau$, respectively, represent normal and shear stresses. The $Q_{i j}(\delta)$ are defined as follows:

$$
\begin{aligned}
Q_{11}(\delta) & =Q_{22}(\delta)=\frac{E(\delta)}{1-\mu^{2}(\delta)}, \\
Q_{12}(\delta) & =\frac{\mu(\delta) E(\delta)}{1-\mu^{2}(\delta)}, \\
Q_{66}(\delta) & =\frac{E(\delta)}{2[1+\mu(\delta)]},
\end{aligned}
$$

$$
U^{i}=\frac{1}{2} \iiint_{V}\left(N_{\varphi}^{i} \varepsilon_{\varphi}^{0, i}+N_{\theta}^{i} \varepsilon_{\theta}^{0, i}+N_{\varphi \theta}^{i} \gamma_{\varphi \theta}^{0, i}+M_{\varphi}^{i} k_{\varphi}^{i}+M_{\theta}^{i} k_{\theta}^{i}+M_{\varphi \theta}^{i} k_{\varphi \theta}^{i}+Q_{\varphi}^{i} \gamma_{\varphi \delta}^{0, i}+Q_{\theta}^{i} \gamma_{\theta \delta}^{0, i}\right) A B \mathrm{~d} \varphi \mathrm{d} \theta \mathrm{d} \delta
$$

where $E$ and $\mu$ are elastic modulus and Poisson's ratio, respectively. The force and moment resultants can be expressed as below:

$$
\begin{aligned}
\left\{\begin{array}{c}
N_{\varphi}^{i} \\
N_{\theta}^{i} \\
N_{\varphi \theta}^{i}
\end{array}\right\}= & {\left[\begin{array}{ccc}
A_{11} & A_{12} & 0 \\
A_{12} & A_{22} & 0 \\
0 & 0 & A_{66}
\end{array}\right]\left\{\begin{array}{c}
\varepsilon_{\varphi}^{0, i} \\
\varepsilon_{\theta}^{0, i} \\
\gamma_{\varphi \theta}^{0, i}
\end{array}\right\} } \\
& +\left[\begin{array}{ccc}
B_{11} & B_{12} & 0 \\
B_{12} & B_{22} & 0 \\
0 & 0 & B_{66}
\end{array}\right]\left\{\begin{array}{c}
\kappa_{\varphi}^{i} \\
\kappa_{\theta}^{i} \\
\kappa_{\varphi \theta}^{i}
\end{array}\right\}, \\
\left\{\begin{array}{c}
M_{\varphi}^{i} \\
M_{\theta}^{i} \\
M_{\varphi \theta}^{i}
\end{array}\right\}= & {\left[\begin{array}{ccc}
B_{11} & B_{12} & 0 \\
B_{12} & B_{22} & 0 \\
0 & 0 & B_{66}
\end{array}\right]\left\{\begin{array}{c}
\varepsilon_{\varphi}^{0, i} \\
\varepsilon_{\theta}^{0, i} \\
\gamma_{\varphi \theta}^{0, i}
\end{array}\right\} } \\
& +\left[\begin{array}{ccc}
D_{11} & D_{12} & 0 \\
D_{12} & D_{22} & 0 \\
0 & 0 & D_{66}
\end{array}\right]\left\{\begin{array}{c}
\kappa_{\varphi}^{i} \\
\kappa_{\theta}^{i} \\
\kappa_{\varphi \theta}^{i}
\end{array}\right\}, \\
\left\{\begin{array}{c}
Q_{\varphi}^{i} \\
Q_{\theta}^{i}
\end{array}\right\}= & \bar{\kappa}\left[\begin{array}{cc}
A_{66} & 0 \\
0 & A_{66}
\end{array}\right]\left[\begin{array}{c}
\gamma_{\varphi \delta}^{0, i} \\
\gamma_{\theta \delta}^{0, i}
\end{array}\right],
\end{aligned}
$$

where $\bar{\kappa}$ represents the shear correction factor, and it is set as $5 / 6$ in this study. The symbols of $A_{i j}, B_{i j}$, and $D_{i j}$ can be written as follows:

$$
\left(A_{i j}, B_{i j}, D_{i j}\right)=\int_{-h / 2}^{h / 2} Q_{i j}(\delta)\left(1, \delta, \delta^{2}\right) \mathrm{d} \delta
$$

The strain energy of the select segment can be expressed as follows: 
The strain energy expression can be rewritten as $U^{i}=U_{S}^{i}+U_{B}^{i}+U_{B C}^{i}$, and the detailed expressions are shown in equations (10)-(12):

$$
\begin{aligned}
U_{S}^{i}= & \frac{1}{2} \iiint_{V}\left\{A_{11}\left(\frac{1}{A} \frac{\partial u^{i}}{\partial \varphi}+\frac{v^{i}}{A B} \frac{\partial A}{\partial \theta}+\frac{w^{i}}{R}\right)^{2}+A_{22}\left(\frac{1}{B} \frac{\partial v^{i}}{\partial \theta}+\frac{u^{i}}{A B} \frac{\partial B}{\partial \varphi}+\frac{w^{i}}{R}\right)^{2}+A_{66}\left(\frac{A}{B} \frac{\partial}{\partial \theta}\left(\frac{u^{i}}{A}\right)+\frac{B}{A} \frac{\partial}{\partial \varphi}\left(\frac{v^{i}}{B}\right)\right)^{2}\right. \\
& \left.+2 A_{12}\left(\frac{1}{A} \frac{\partial u^{i}}{\partial \varphi}+\frac{v^{i}}{A B} \frac{\partial A}{\partial \theta}+\frac{w^{i}}{R}\right)\left(\frac{1}{B} \frac{\partial v^{i}}{\partial \theta}+\frac{u^{i}}{A B} \frac{\partial B}{\partial \varphi}+\frac{w^{i}}{R}\right)+\bar{\kappa} A_{66}\left(\frac{1}{A} \frac{\partial w^{i}}{\partial \varphi}-\frac{u^{i}}{R}+\psi_{\varphi}^{i}\right)^{2}+\bar{\kappa} A_{66}\left(\frac{1}{B} \frac{\partial w^{i}}{\partial \theta}-\frac{v^{i}}{R}+\psi_{\theta}^{i}\right)^{2}\right\} A B \mathrm{~d} \varphi \mathrm{d} \theta \mathrm{d} \delta
\end{aligned}
$$

$$
\begin{aligned}
U_{B}^{i}= & \frac{1}{2} \iiint_{V}\left\{D_{11}\left(\frac{1}{A} \frac{\partial \psi_{\varphi}^{i}}{\partial \varphi}+\frac{\psi_{\theta}^{i}}{A B} \frac{\partial A}{\partial \theta}\right)^{2}+D_{22}\left(\frac{1}{B} \frac{\partial \psi_{\theta}^{i}}{\partial \theta}+\frac{\psi_{\varphi}^{i}}{A B} \frac{\partial B}{\partial \varphi}\right)^{2}+D_{66}\left(\frac{A}{B} \frac{\partial}{\partial \theta}\left(\frac{\psi_{\varphi}^{i}}{A}\right)+\frac{B}{A} \frac{\partial}{\partial \varphi}\left(\frac{\psi_{\theta}^{i}}{B}\right)\right)^{2}\right. \\
& \left.+2 D_{12}\left(\frac{1}{A} \frac{\partial \psi_{\varphi}^{i}}{\partial \varphi}+\frac{\psi_{\theta}^{i}}{A B} \frac{\partial A}{\partial \theta}\right)\left(\frac{1}{B} \frac{\partial \psi_{\theta}^{i}}{\partial \theta}+\frac{\psi_{\varphi}^{i}}{A B} \frac{\partial B}{\partial \varphi}\right)\right\} A B \mathrm{~d} \varphi \mathrm{d} \theta \mathrm{d} \delta
\end{aligned}
$$

$U_{B S}^{i}=\iiint_{V}\left\{B_{11}\left(\frac{1}{A} \frac{\partial u^{i}}{\partial \varphi}+\frac{v^{i}}{A B} \frac{\partial A}{\partial \theta}+\frac{w^{i}}{R}\right)\left(\frac{1}{A} \frac{\partial \psi_{\varphi}^{i}}{\partial \varphi}+\frac{\psi_{\theta}^{i}}{A B} \frac{\partial A}{\partial \theta}\right)+B_{12}\left(\frac{1}{A} \frac{\partial u^{i}}{\partial \varphi}+\frac{v^{i}}{A B} \frac{\partial A}{\partial \theta}+\frac{w^{i}}{R}\right)\left(\frac{1}{B} \frac{\partial \psi_{\theta}^{i}}{\partial \theta}+\frac{\psi_{\varphi}^{i}}{A B} \frac{\partial B}{\partial \varphi}\right)\right.$

$$
\begin{aligned}
& +B_{12}\left(\frac{1}{B} \frac{\partial v^{i}}{\partial \theta}+\frac{u^{i}}{A B} \frac{\partial B}{\partial \varphi}+\frac{w^{i}}{R}\right)\left(\frac{1}{A} \frac{\partial \psi_{\varphi}^{i}}{\partial \varphi}+\frac{\psi_{\theta}^{i}}{A B} \frac{\partial A}{\partial \theta}\right)+B_{66}\left(\frac{1}{A} \frac{\partial u^{i}}{\partial \varphi}+\frac{v^{i}}{A B} \frac{\partial A}{\partial \theta}+\frac{w^{i}}{R}\right)\left(\frac{A}{B} \frac{\partial}{\partial \theta}\left(\frac{\psi_{\varphi}^{i}}{A}\right)+\frac{B}{A} \frac{\partial}{\partial \varphi}\left(\frac{\psi_{\theta}^{i}}{B}\right)\right) \\
& \left.+B_{22}\left(\frac{1}{B} \frac{\partial v^{i}}{\partial \theta}+\frac{u^{i}}{A B} \frac{\partial B}{\partial \varphi}+\frac{w^{i}}{R}\right)\left(\frac{1}{B} \frac{\partial \psi_{\theta}^{i}}{\partial \theta}+\frac{\psi_{\varphi}^{i}}{A B} \frac{\partial B}{\partial \varphi}\right)\right\} A B \mathrm{~d} \varphi \mathrm{d} \theta \mathrm{d} \delta
\end{aligned}
$$
follows:

The kinetic energy of the select segment can be written as

$$
\begin{aligned}
T^{i} & =\frac{1}{2} \iiint_{V} \rho(\delta)\left[\left(\dot{\bar{U}}^{i}\right)^{2}+\left(\dot{\bar{V}}^{i}\right)^{2}+\left(\dot{\bar{W}}^{i}\right)^{2}\right]\left(1+\frac{\delta}{R}\right)^{2} A B \mathrm{~d} \varphi \mathrm{d} \theta \mathrm{d} \delta \\
& =\frac{1}{2} \int_{\varphi_{0}}^{\varphi_{1}} \int_{0}^{2 \pi}\left\{I_{0}\left[\left(\dot{u}^{i}\right)^{2}+\left(\dot{v}^{i}\right)^{2}+\left(\dot{w}^{i}\right)^{2}\right]+2 I_{1}\left(\dot{u}^{i} \dot{\psi}_{\varphi}^{i}+\dot{v}^{i} \dot{\psi}_{\theta}^{i}\right)+I_{2}\left[\left(\dot{\psi}_{\varphi}^{i}\right)^{2}+\left(\dot{\psi}_{\theta}^{i}\right)^{2}\right]\right\} A B \mathrm{~d} \varphi \mathrm{d} \theta
\end{aligned}
$$

where the dot on the symbols represents differentiation about time, and the related symbols can be expressed as follows:

$$
\left(I_{0}, I_{1}, I_{2}\right)=\int_{-h / 2}^{h / 2} \rho(\delta)\left(1+\frac{\delta}{R}\right)^{2}\left(1, \delta, \delta^{2}\right) \mathrm{d} \delta
$$

As mentioned above, the application of artificial springs can ensure the convergence of result. Each edge of the structure is restrained by three linear springs $\left(k_{u}, k_{v}, k_{w}\right)$ and two rotational springs $\left(k_{\varphi}, k_{\theta}\right)$. Then, the boundary potential energy for moderately thick annular spherical shell is expressed as follows: 


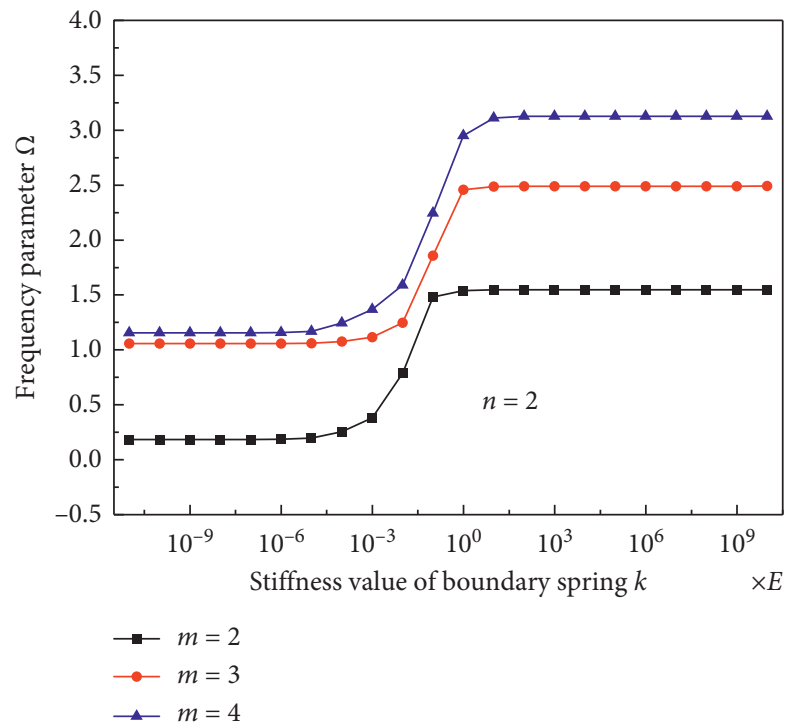

(a)

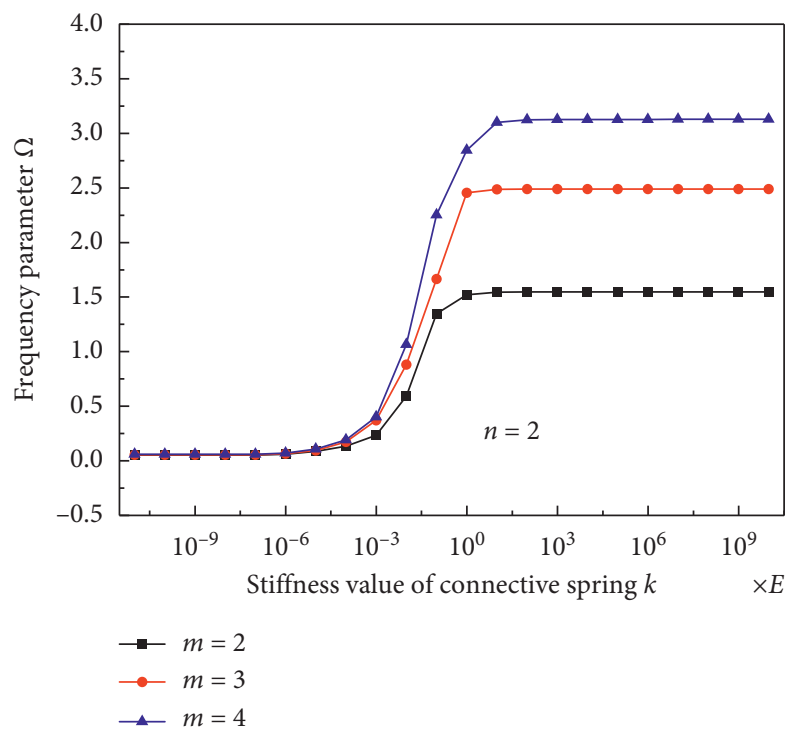

(c)

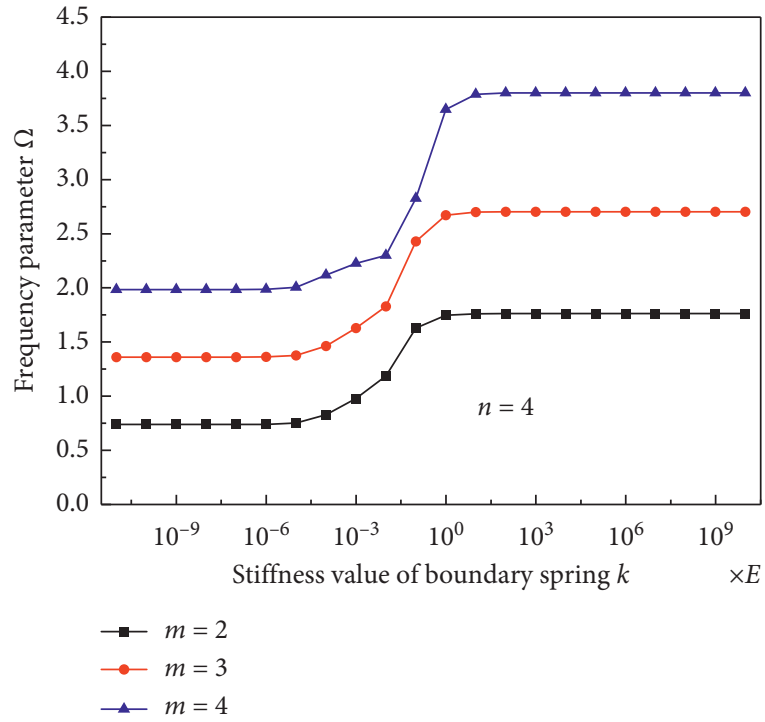

(b)

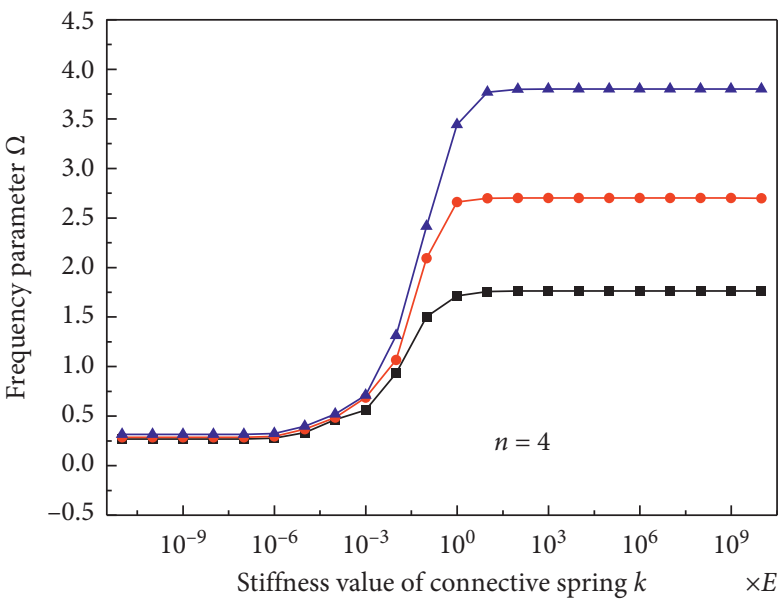

$\rightarrow m=2$

$\longrightarrow m=3$

$\_m=4$

Figure 2: Frequency parameters $\Omega$ of the annular spherical shell with different boundary parameters.

$$
\begin{aligned}
U_{b}= & \frac{1}{2} \int_{0}^{2 \pi} \int_{-h / 2}^{h / 2}\left\{k_{u, 0} u^{2}+k_{v, 0} v^{2}+k_{w, 0} w^{2}+k_{\varphi, 0} \psi_{\varphi}^{2}+k_{\theta, 0} \psi_{\theta}^{2}\right\}_{x=x_{l, 0}} B \mathrm{~d} \theta \mathrm{d} \delta \\
& +\frac{1}{2} \int_{0}^{2 \pi} \int_{-h / 2}^{h / 2}\left\{k_{u, 1} u^{2}+k_{v, 1} v^{2}+k_{w, 1} w^{2}+k_{\varphi, 1} \psi_{\varphi}^{2}+k_{\theta, 1} \psi_{\theta}^{2}\right\}_{x=x_{l, 1}} B \mathrm{~d} \theta \mathrm{d} \delta,
\end{aligned}
$$

where $k_{t, 0}(t=u, v, w, \varphi, \theta)$ and $k_{t, 1}$, respectively, denote the spring stiffness value of the moderately thick annular spherical shell.

The potential energy stored in two adjacent segments can be shown as follows:

$$
\begin{aligned}
U_{s}^{i}= & \frac{1}{2} \int_{0}^{2 \pi} \int_{-h / 2}^{h / 2}\left\{k_{u}\left(u^{i}-u^{i+1}\right)^{2}+k_{v}\left(v^{i}-v^{i+1}\right)^{2}+k_{w}\left(w^{i}-w^{i+1}\right)^{2}\right. \\
& \left.+k_{\varphi}\left(\psi_{\varphi}^{i}-\psi_{\varphi}^{i+1}\right)^{2}+k_{\theta}\left(\psi_{\theta}^{i}-\psi_{\theta}^{i+1}\right)^{2}\right\}_{i, i+1} B \mathrm{~d} \theta \mathrm{d} \delta .
\end{aligned}
$$


TABLE 1: Related spring stiffness values.

\begin{tabular}{lcccc}
\hline BC & $k_{u, 0}, k_{u, 1}$ & $k_{v, 0}, k_{v, 1}$ & $k_{w, 0}, k_{w, 1}$ & $k_{\varphi, 0}, k_{\varphi, 1}$ \\
\hline$F$ & 0 & 0 & 0 & 0 \\
SD & 0 & $10^{4} E$ & $10^{4} E$ & 0 \\
SS & $10^{4} E$ & $10^{4} E$ & $10^{4} E$ & 0 \\
$C$ & $10^{4} E$ & $10^{4} E$ & $10^{4} E$ & 0 \\
$E 1$ & $10^{-1} E$ & $10^{4} E$ & $10^{4} E$ & $10^{4} E$ \\
$E 2$ & $10^{4} E$ & $10^{-1} E$ & $10^{4} E$ & $10^{4} E$ \\
$E 3$ & $10^{-1} E$ & $10^{-1} E$ & $10^{4} E$ & $10^{4} E$ \\
\hline
\end{tabular}

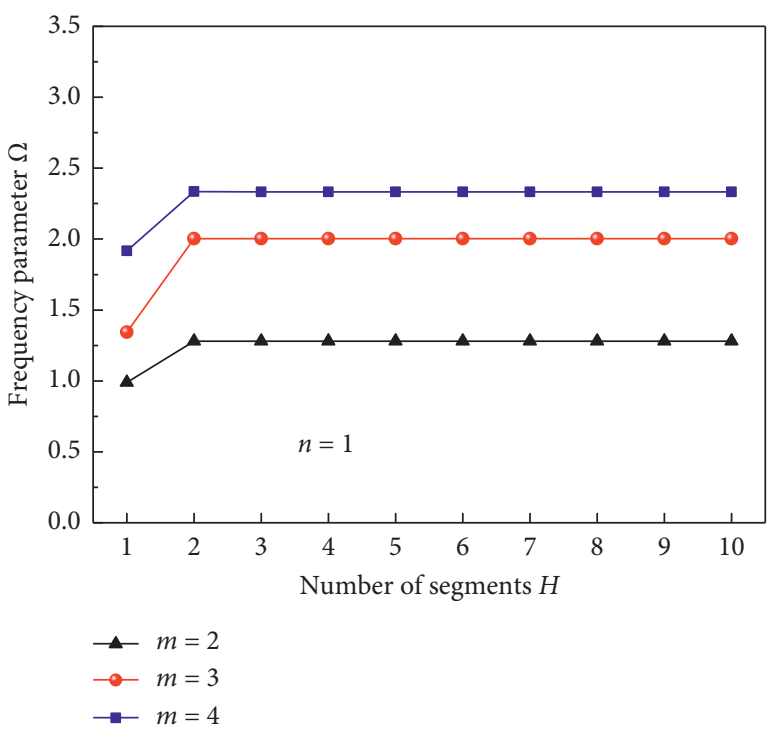

(a)

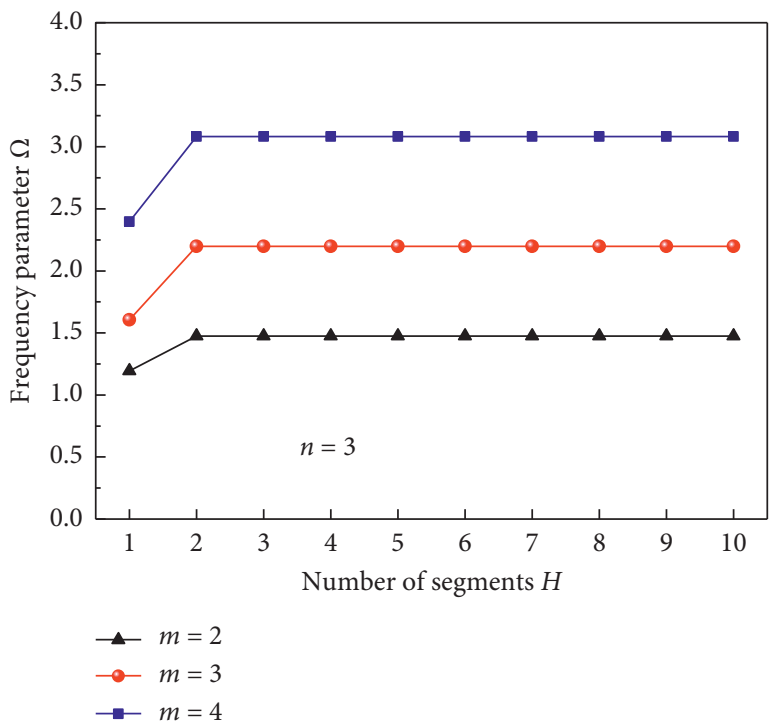

(c)

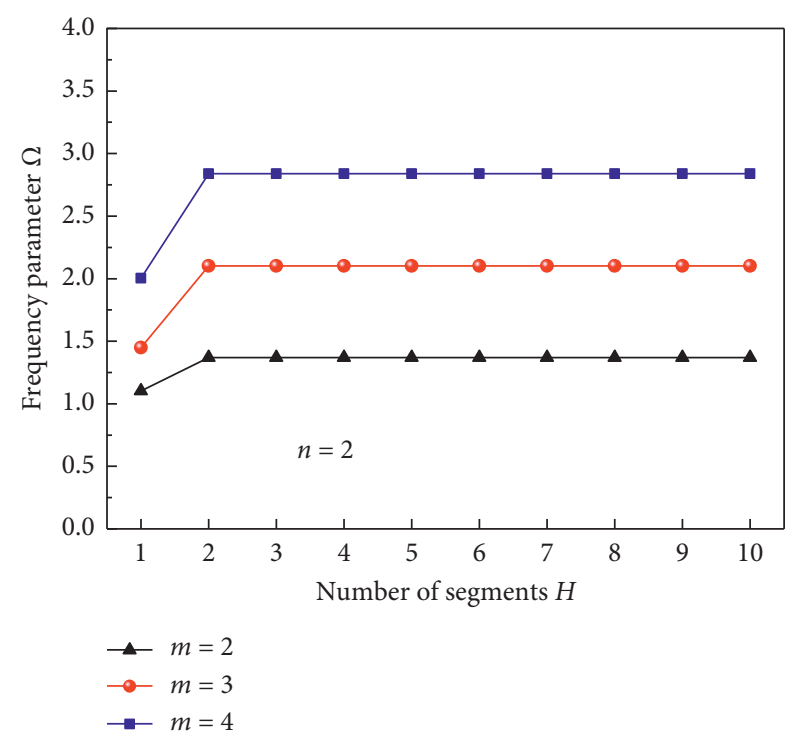

(b)

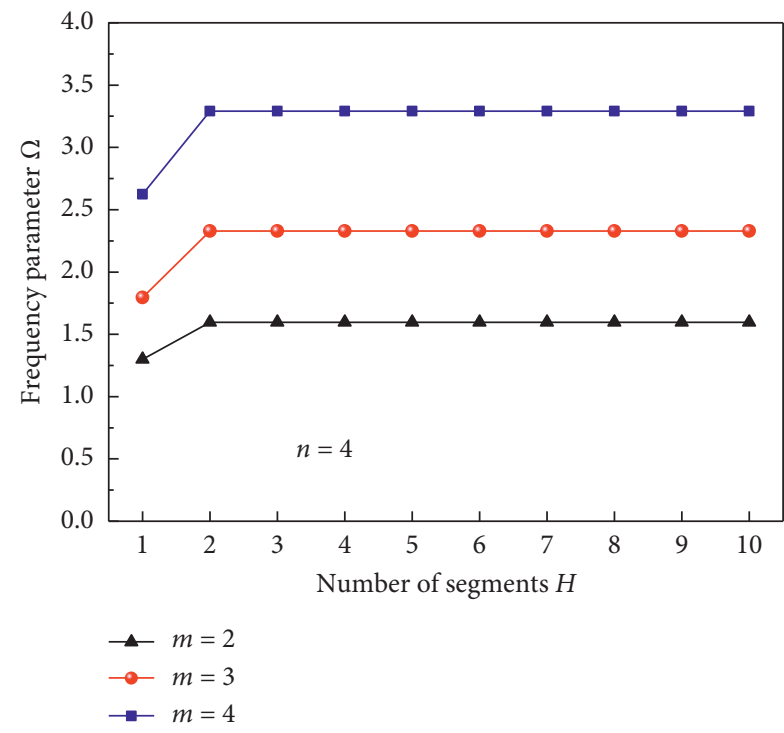

(d)

FIGURE 3: Frequency parameters $\Omega$ of the annular spherical shell with different number of segments.

Thus, the total potential energy can be written as follows:

$$
U_{B S}=U_{b}+\sum_{i=1}^{I-1} U_{s}^{i} .
$$

2.3. Admissible Displacement Functions. In this paper, the authors try to introduce the unified Jacobi polynomials to improve the selection of displacement functions based on the domain decomposition method, and the value of $\varphi$ are 


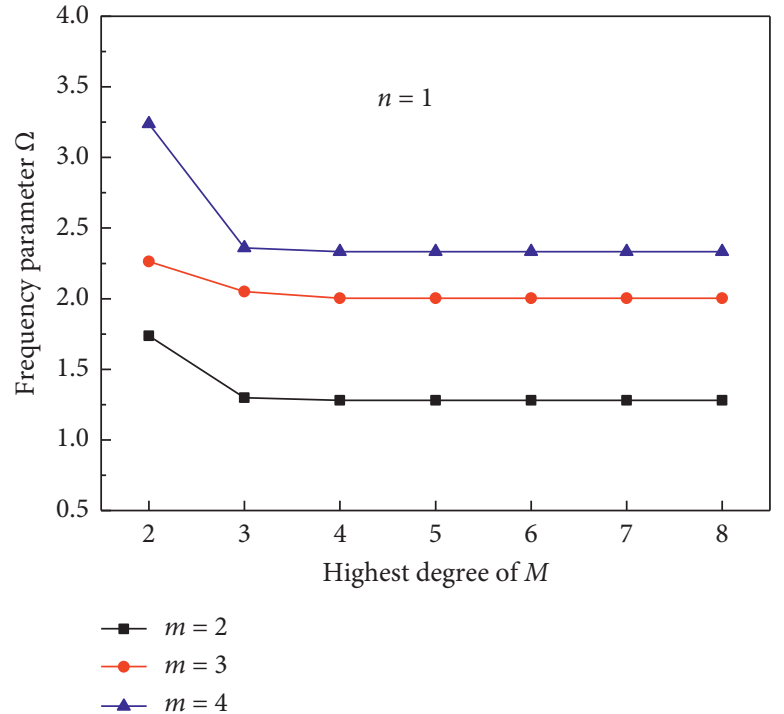

(a)

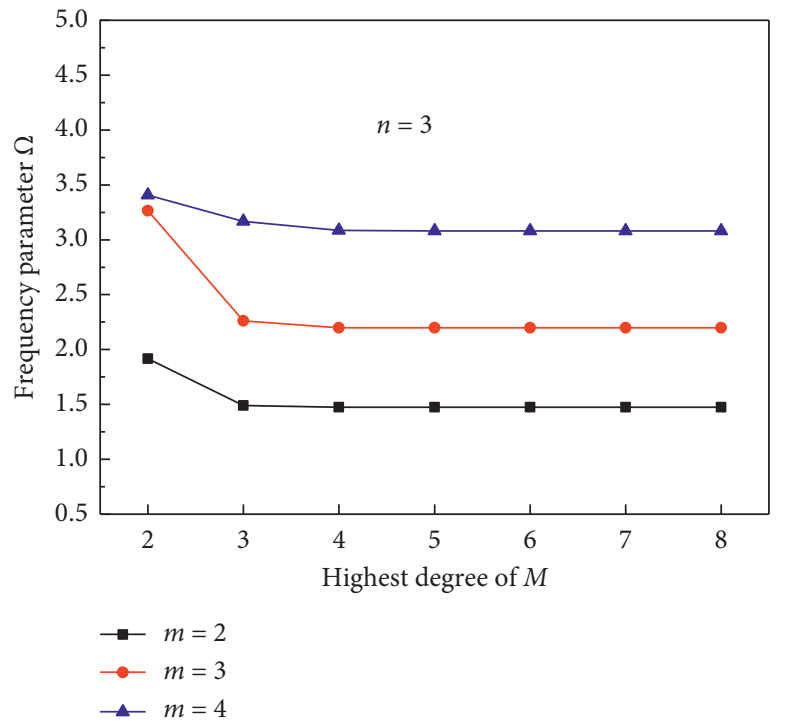

(c)

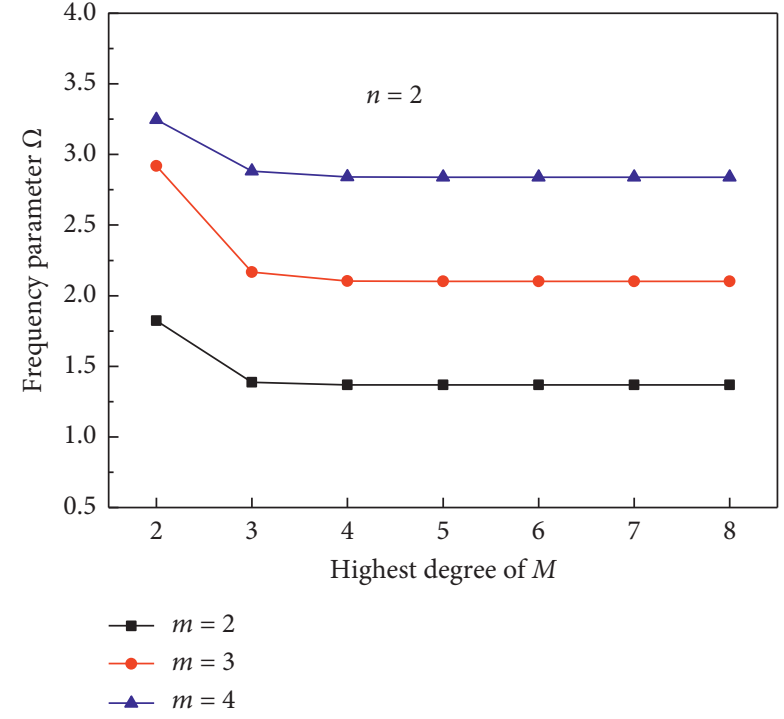

(b)

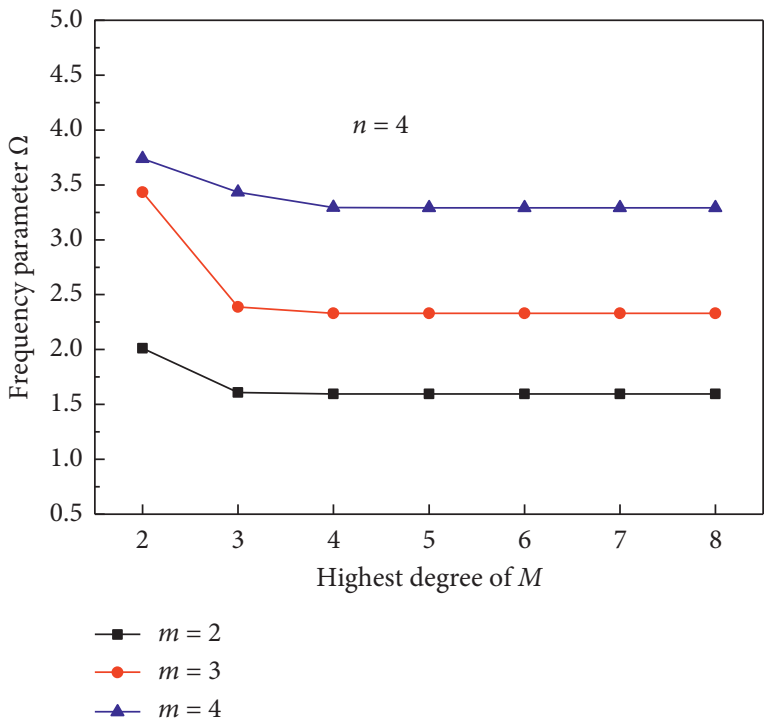

(d)

FIGURE 4: Frequency parameters $\Omega$ of the annular spherical shell with different truncation.
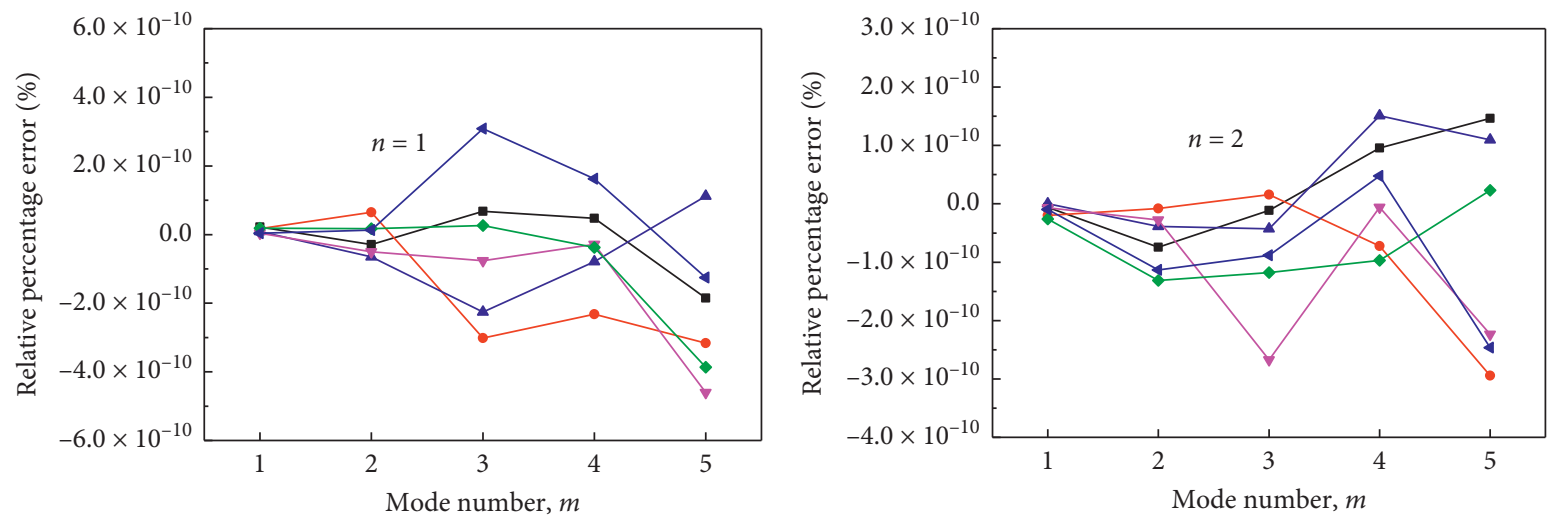

$$
\begin{array}{ll}
\longrightarrow \alpha=-0.5 \beta=-0.5 & \longrightarrow \alpha=0 \beta=0 \\
\longrightarrow \alpha=0.5 \beta=0 & \longrightarrow \alpha=1 \beta=1 \\
\longrightarrow \alpha=-0.5 \beta=0 & \longrightarrow \alpha=1 \beta=0.5
\end{array}
$$

(a)

$$
\begin{aligned}
& \rightarrow \alpha=-0.5 \beta=-0.5 \quad \rightarrow \alpha=0 \beta=0 \\
& \neg \alpha=0.5 \beta=0 \quad \neg \alpha=1 \beta=1 \\
& \rightarrow \alpha=-0.5 \beta=0 \quad \neg \alpha=1 \beta=0.5
\end{aligned}
$$

Figure 5: Continued. 


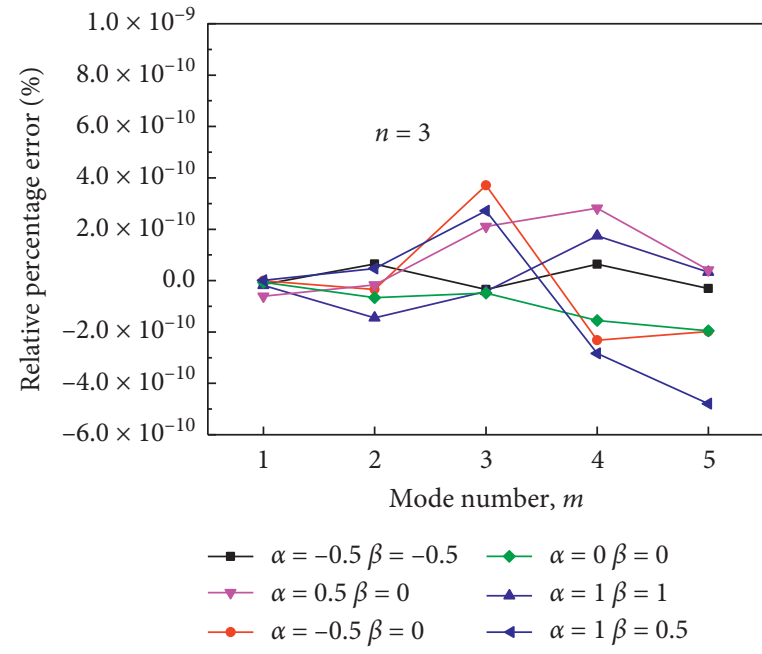

(c)

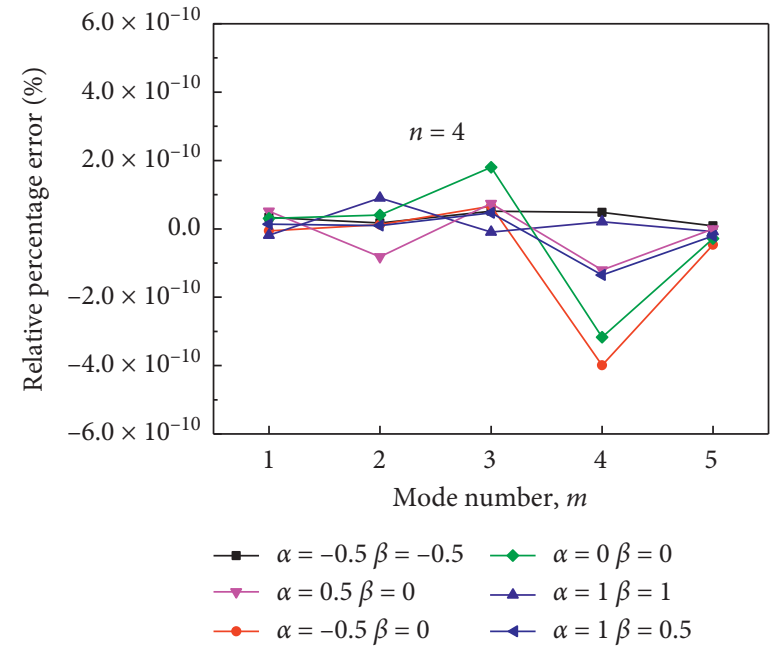

(d)

FIGURE 5: Relative percentage error of the annular spherical shell with different Jacobi parameters.

defined in the range of $[-1,1]$. The related recurrence formulas are shown as below:

$$
\begin{aligned}
P_{0}^{(\alpha, \beta)}(\phi)= & 1, \\
P_{1}^{(\alpha, \beta)}(\phi)= & \frac{\alpha+\beta+2}{2} \phi-\frac{\alpha-\beta}{2}, \\
P_{i}^{(\alpha, \beta)}(\phi)= & \frac{(\alpha+\beta+2 i-1)\left\{\alpha^{2}-\beta^{2}+\phi(\alpha+\beta+2 i)(\alpha+\beta+2 i-2)\right\}}{2 i(\alpha+\beta+i)(\alpha+\beta+2 i-2)} P_{i-1}^{(\alpha, \beta)}(\phi) \\
& -\frac{(\alpha+i-1)(\beta+i-1)(\alpha+\beta+2 i)}{i(\alpha+\beta+i)(\alpha+\beta+2 i-2)} P_{i-2}^{(\alpha, \beta)}(\phi),
\end{aligned}
$$

where $\alpha, \beta>-1$ and $i=2,3, \ldots$

Therefore, the displacement functions can be generalized according to Jacobi polynomial:

$$
\begin{gathered}
u=\sum_{m=0}^{M} U_{m} P_{m}^{(\alpha, \beta)}(\varphi) \cos (n \theta) e^{i \omega t}, \\
v=\sum_{m=0}^{M} V_{m} P_{m}^{(\alpha, \beta)}(\varphi) \sin (n \theta) e^{i \omega t}, \\
w=\sum_{m=0}^{M} W_{m} P_{m}^{(\alpha, \beta)}(\varphi) \cos (n \theta) e^{i \omega t}, \\
\psi_{x}=\sum_{m=0}^{M} \psi_{x m} P_{m}^{(\alpha, \beta)}(\varphi) \cos (n \theta) e^{i \omega t}, \\
\psi_{\theta}=\sum_{m=0}^{M} \psi_{\theta m} P_{m}^{(\alpha, \beta)}(\varphi) \cos (n \theta) e^{i \omega t},
\end{gathered}
$$

where $U_{m}, V_{m}, W_{m}, \psi_{\theta m}$, and $\psi_{\varphi m}$ are unknown coefficients; $m$ and $n$, respectively, represent the semiwave number in axial and circumferential direction, and $M$ is highest degrees of $m$.

2.4. Solution Procedure. The Lagrangian energy functions $\mathscr{L}$ can be expressed as follows:

$$
\mathscr{L}=\sum_{i=1}^{H}\left(T^{i}-U^{i}\right)-U_{B S}
$$

Differentiate with regard to undetermined coefficients, and the formula can be obtained:

$$
\begin{aligned}
\frac{\partial \mathscr{L}}{\partial \vartheta} & =0, \\
\vartheta & =U_{m}, V_{m}, W_{m}, \psi_{x m}, \psi_{\theta m} .
\end{aligned}
$$

Substituting the formulas mentioned above into equation (21), the matrix form can be shown as follows: 
TABLE 2: Comparison of frequency for the annular spherical shell.

\begin{tabular}{|c|c|c|c|c|c|c|c|c|c|}
\hline \multirow{2}{*}{$n$} & \multirow{2}{*}{$m$} & \multicolumn{2}{|c|}{$\mathrm{F}-\mathrm{F}$} & \multicolumn{2}{|c|}{$\mathrm{F}-\mathrm{C}$} & \multicolumn{2}{|c|}{$\mathrm{C}-\mathrm{F}$} & \multicolumn{2}{|c|}{$\mathrm{C}-\mathrm{C}$} \\
\hline & & Present & FEM & Present & FEM & Present & FEM & Present & FEM \\
\hline \multirow{5}{*}{2} & 1 & 58.34 & 58.37 & 335.44 & 335.69 & 158.55 & 158.70 & 1067.35 & 1068.10 \\
\hline & 2 & 157.40 & 157.67 & 977.91 & 978.15 & 932.69 & 932.60 & 1335.74 & 1337.50 \\
\hline & 3 & 912.75 & 912.59 & 1389.15 & 1391.20 & 1336.83 & 1339.10 & 2150.97 & 2158.90 \\
\hline & 4 & 996.66 & 996.95 & 1856.99 & 1858.60 & 1562.82 & 1564.30 & 2701.76 & 2707.30 \\
\hline & 5 & 1455.59 & 1456.90 & 2251.48 & 2259.40 & 2132.67 & 2145.30 & 2992.14 & 3001.10 \\
\hline \multirow{5}{*}{3} & 1 & 160.40 & 160.48 & 384.62 & 385.04 & 176.77 & 176.88 & 1031.73 & 1032.30 \\
\hline & 2 & 385.48 & 386.04 & 1068.80 & 1069.70 & 1004.08 & 1004.50 & 1421.22 & 1423.40 \\
\hline & 3 & 1065.40 & 1066.10 & 1515.69 & 1518.30 & 1453.91 & 1456.30 & 2232.02 & 2240.20 \\
\hline & 4 & 1380.75 & 1382.20 & 2111.14 & 2113.00 & 1788.38 & 1789.70 & 3024.52 & 3032.60 \\
\hline & 5 & 1576.96 & 1578.60 & 2354.72 & 2363.20 & 2282.17 & 2290.50 & 3351.62 & 3372.60 \\
\hline \multirow{5}{*}{4} & 1 & 294.15 & 294.35 & 621.79 & 622.71 & 297.93 & 298.13 & 1055.37 & 1055.90 \\
\hline & 2 & 636.24 & 637.31 & 1153.80 & 1155.10 & 1073.02 & 1073.80 & 1521.82 & 1524.40 \\
\hline & 3 & 1174.39 & 1175.80 & 1664.41 & 1667.90 & 1571.91 & 1574.60 & 2333.59 & 2342.00 \\
\hline & 4 & 1713.64 & 1717.30 & 2483.20 & 2490.80 & 2155.14 & 2156.90 & 3283.18 & 3295.50 \\
\hline & 5 & 1960.10 & 1961.80 & 2596.72 & 2601.80 & 2398.24 & 2406.60 & 3483.08 & 3499.60 \\
\hline \multirow{5}{*}{5} & 1 & 453.10 & 453.55 & 888.92 & 890.55 & 454.66 & 455.10 & 1128.99 & 1129.70 \\
\hline & 2 & 905.27 & 907.10 & 1276.79 & 1278.80 & 1172.80 & 1173.90 & 1645.58 & 1648.50 \\
\hline & 3 & 1325.75 & 1328.00 & 1840.65 & 1845.10 & 1714.25 & 1717.40 & 2464.25 & 2472.80 \\
\hline & 4 & 1908.15 & 1912.73 & 2664.28 & 2673.60 & 2545.11 & 2553.10 & 3497.85 & 3518.60 \\
\hline & 5 & 2509.94 & 2513.10 & 3175.94 & 3182.60 & 2595.33 & 2598.80 & 3781.52 & 3790.10 \\
\hline
\end{tabular}

TABLE 3: Comparison of the results for the annular spherical shell $(m=1)$.

\begin{tabular}{|c|c|c|c|c|c|c|c|c|}
\hline \multirow{2}{*}{$n$} & \multicolumn{2}{|c|}{$\mathrm{F}-\mathrm{F}$} & \multicolumn{2}{|c|}{$\mathrm{F}-\mathrm{C}$} & \multicolumn{2}{|c|}{$\mathrm{C}-\mathrm{F}$} & \multicolumn{2}{|c|}{$\mathrm{C}-\mathrm{C}$} \\
\hline & Present & Qu et al. [37] & Present & Qu et al. [37] & Present & Qu et al. [37] & Present & Qu et al. [37] \\
\hline 0 & 0.86794 & 0.87054 & 0.81696 & 0.81661 & 0.40539 & 0.40549 & 0.98744 & 0.98710 \\
\hline 1 & 0.86154 & 0.86412 & 0.60870 & 0.60880 & 0.15171 & 0.15170 & 1.02673 & 1.02639 \\
\hline 2 & 0.05724 & 0.05726 & 0.34849 & 0.34857 & 0.07635 & 0.07636 & 1.00321 & 1.00292 \\
\hline 3 & 0.15319 & 0.15323 & 0.65740 & 0.65733 & 0.15408 & 0.15412 & 0.99835 & 0.99803 \\
\hline 4 & 0.27893 & 0.27900 & 0.96571 & 0.96537 & 0.27901 & 0.27908 & 1.04307 & 1.04274 \\
\hline 5 & 0.42932 & 0.42940 & 1.11593 & 1.11557 & 0.42933 & 0.42941 & 1.12659 & 1.12623 \\
\hline 6 & 0.60179 & 0.60188 & 1.24443 & 1.24402 & 0.60180 & 0.60189 & 1.24619 & 1.24578 \\
\hline 7 & 0.79550 & 0.79559 & 1.40073 & 1.40026 & 0.79550 & 0.79559 & 1.40104 & 1.40056 \\
\hline 8 & 1.01041 & 1.01048 & 1.58989 & 1.58934 & 1.01041 & 1.01048 & 1.58994 & 1.58939 \\
\hline 9 & 1.24667 & 1.24672 & 1.81090 & 1.81026 & 1.24667 & 1.24672 & 1.81091 & 1.81027 \\
\hline
\end{tabular}

$$
\left(\mathrm{K}-\omega^{2} \mathrm{M}\right) \mathrm{Q}=0,
$$

where $\mathbf{K}, \mathbf{M}$, and $\mathbf{Q}$ of equation (22) represent stiffness matrix, mass matrix, and unknown coefficients matrix.

\section{Numerical Results and Discussion}

According to the above research, a unified analytical model for moderately thick annular spherical shell is established. The main purpose of this section is to discuss the numerical results. According to the experience of published literatures, the various boundary conditions can be represented by the first letter of a word. For example, the free and elastic edge conditions are denoted by the letter $F$ and $E_{i}(i=1,2,3)$. The material properties are chosen as $E=168 \mathrm{GPa}, \rho=$ $5700 \mathrm{~kg} / \mathrm{m}^{3}$, and $\mu=0.3$. In addition, other related parameters appeared in this paper are defined as $M=8, \alpha=\beta=0.5$,
$H=6 ; R=1 \mathrm{~m}, \varphi_{0}=\pi / 6, \varphi_{1}=\pi / 2$, and $h=0.06 \mathrm{~m}$. The results of this paper are handle by $\Omega=\omega R \sqrt{\rho / E}$.

3.1. Convergence Study. In order to investigate the convergence of different spring stiffness values, Figure 2 illustrates the frequency parameters of moderately thick annular spherical shell with different boundary parameters. No matter for boundary spring and connective spring, it is obvious that the edge condition changes from free to clamped case with the spring stiffness values in range of $10^{-11} \mathrm{E}$ to $10^{10} \mathrm{E}$. For example, the boundary condition is clamped when spring stiffness values are defined in $10^{3} \mathrm{E}$ to $10^{10} \mathrm{E}$. Thus, the various boundary conditions can be easily obtained through the analysis. All edge conditions used in this study are shown in Table 1.

Figure 3 shows results of moderately thick annular spherical shell with different segment numbers, and the 
TABLE 4: Results of the annular spherical shell with classical boundary conditions $\left(\varphi_{0}=\pi / 6, \varphi_{1}=\pi / 2, h=0.06 \mathrm{~m}\right.$, and $\left.R=1 \mathrm{~m}\right)$.

\begin{tabular}{|c|c|c|c|c|c|c|c|c|c|c|}
\hline \multirow{2}{*}{$n$} & \multirow{2}{*}{$m$} & \multicolumn{9}{|c|}{ Boundary conditions } \\
\hline & & $\mathrm{F}-\mathrm{C}$ & $\mathrm{F}-\mathrm{SS}$ & F-SD & $\mathrm{C}-\mathrm{C}$ & $\mathrm{C}-\mathrm{SS}$ & $\mathrm{C}-\mathrm{SD}$ & SD-SD & SD-SS & SS-SS \\
\hline \multirow{5}{*}{1} & 1 & 0.6817 & 0.6506 & 0.3576 & 1.2726 & 1.1611 & 0.7320 & 0.6097 & 1.0460 & 1.0823 \\
\hline & 2 & 0.9894 & 0.9577 & 0.7359 & 1.2795 & 1.2727 & 1.1643 & 1.0542 & 1.1558 & 1.2720 \\
\hline & 3 & 1.3427 & 1.2516 & 0.7955 & 2.0030 & 1.8598 & 1.5264 & 1.2667 & 1.7017 & 1.7203 \\
\hline & 4 & 1.9047 & 1.8469 & 1.1163 & 2.3335 & 2.3046 & 1.8602 & 1.7053 & 2.1163 & 2.2841 \\
\hline & 5 & 2.1354 & 2.1184 & 1.4217 & 2.8789 & 2.7077 & 2.5767 & 2.1325 & 2.3296 & 2.5383 \\
\hline \multirow{5}{*}{2} & 1 & 0.3605 & 0.3382 & 0.2278 & 1.1451 & 1.1290 & 0.9540 & 0.9382 & 1.0524 & 1.1290 \\
\hline & 2 & 1.1020 & 1.0643 & 0.9936 & 1.3688 & 1.2626 & 1.2556 & 1.1866 & 1.1893 & 1.1957 \\
\hline & 3 & 1.4457 & 1.3421 & 1.1197 & 2.1017 & 1.9256 & 1.5831 & 1.3930 & 1.7653 & 1.7855 \\
\hline & 4 & 1.9971 & 1.9560 & 1.3690 & 2.8395 & 2.7567 & 1.9251 & 1.7650 & 2.4661 & 2.5941 \\
\hline & 5 & 2.2387 & 2.1109 & 2.0135 & 3.1365 & 3.0247 & 2.8368 & 2.6382 & 2.7026 & 2.9972 \\
\hline \multirow{5}{*}{3} & 1 & 0.5538 & 0.5497 & 0.5485 & 1.1221 & 1.1032 & 1.0197 & 0.9967 & 1.0613 & 1.0987 \\
\hline & 2 & 1.1934 & 1.1543 & 1.0763 & 1.4746 & 1.3692 & 1.3590 & 1.2831 & 1.3021 & 1.3158 \\
\hline & 3 & 1.6014 & 1.4856 & 1.4830 & 2.1979 & 2.0213 & 1.9902 & 1.8637 & 1.8746 & 1.8970 \\
\hline & 4 & 2.3367 & 2.1670 & 1.8228 & 3.0816 & 2.9144 & 2.0751 & 1.9907 & 2.7311 & 2.7447 \\
\hline & 5 & 2.4780 & 2.4739 & 2.1649 & 3.3585 & 3.3122 & 2.9481 & 2.7569 & 3.0208 & 3.3013 \\
\hline \multirow{5}{*}{4} & 1 & 0.8911 & 0.8884 & 0.8831 & 1.1667 & 1.1417 & 1.0872 & 1.0724 & 1.1201 & 1.1360 \\
\hline & 2 & 1.2970 & 1.2470 & 1.1855 & 1.5956 & 1.4920 & 1.4687 & 1.4051 & 1.4324 & 1.4501 \\
\hline & 3 & 1.7894 & 1.6701 & 1.6583 & 2.3293 & 2.1575 & 2.1439 & 2.0165 & 2.0296 & 2.0533 \\
\hline & 4 & 2.5440 & 2.3725 & 2.3567 & 3.2899 & 3.0849 & 2.6580 & 2.6352 & 2.9121 & 2.9279 \\
\hline & 5 & 3.1451 & 3.1322 & 2.5656 & 3.7065 & 3.7013 & 3.0904 & 2.9122 & 3.5558 & 3.6988 \\
\hline
\end{tabular}

TABLE 5: Results of annular spherical shell with elastic boundary conditions $(\varphi 0=\pi / 6, \varphi 1=\pi / 2, h=0.06 \mathrm{~m}$, and $R=1 \mathrm{~m})$.

\begin{tabular}{|c|c|c|c|c|c|c|c|}
\hline \multirow{2}{*}{$n$} & \multirow{2}{*}{$m$} & \multicolumn{6}{|c|}{ Boundary conditions } \\
\hline & & E1-E1 & E1-E2 & E1-E3 & $\mathrm{E} 2-\mathrm{E} 2$ & E2-E3 & E3-E3 \\
\hline \multirow{5}{*}{1} & 1 & 0.6682 & 0.8025 & 0.4156 & 0.6744 & 0.4500 & 0.1894 \\
\hline & 2 & 1.1437 & 1.2778 & 0.9634 & 1.2766 & 0.9529 & 0.4576 \\
\hline & 3 & 1.3670 & 1.5104 & 1.3662 & 1.6952 & 1.3363 & 1.2948 \\
\hline & 4 & 2.0209 & 2.0138 & 1.5122 & 2.1306 & 1.7902 & 1.4920 \\
\hline & 5 & 2.1526 & 2.3845 & 2.0636 & 2.2725 & 2.1369 & 2.0342 \\
\hline \multirow{5}{*}{2} & 1 & 0.9770 & 0.9395 & 0.6613 & 0.9195 & 0.6564 & 0.6201 \\
\hline & 2 & 1.3532 & 1.3533 & 1.3517 & 1.3495 & 1.3495 & 0.7010 \\
\hline & 3 & 1.4163 & 2.0186 & 1.3672 & 2.0245 & 1.4248 & 1.3519 \\
\hline & 4 & 2.0912 & 2.1530 & 2.0360 & 2.3291 & 2.0251 & 2.0211 \\
\hline & 5 & 2.7441 & 2.8315 & 2.3495 & 2.7795 & 2.4778 & 2.2574 \\
\hline \multirow{5}{*}{3} & 1 & 1.0410 & 1.0240 & 0.8554 & 1.0209 & 0.8517 & 0.8132 \\
\hline & 2 & 1.4542 & 1.4424 & 1.4052 & 1.4412 & 1.4061 & 1.2166 \\
\hline & 3 & 1.9933 & 2.1648 & 1.8316 & 2.1619 & 1.8503 & 1.6942 \\
\hline & 4 & 2.1833 & 2.6963 & 2.1766 & 2.7773 & 2.1761 & 2.1124 \\
\hline & 5 & 3.1657 & 3.1378 & 3.1087 & 3.1375 & 3.1308 & 2.8808 \\
\hline \multirow{5}{*}{4} & 1 & 1.1213 & 1.1154 & 1.0133 & 1.1150 & 1.0126 & 0.9892 \\
\hline & 2 & 1.5674 & 1.5626 & 1.5162 & 1.5626 & 1.5167 & 1.4476 \\
\hline & 3 & 2.3086 & 2.3037 & 2.2259 & 2.3033 & 2.2256 & 2.2244 \\
\hline & 4 & 2.6444 & 3.2899 & 2.4510 & 3.2893 & 2.4567 & 2.3376 \\
\hline & 5 & 3.3018 & 3.3037 & 3.2906 & 3.3361 & 3.2898 & 3.2623 \\
\hline
\end{tabular}

boundary condition is set as clamped-clamped case. The example shows clearly that the current method converges quickly with the increase of $H$, that is to say, it is not necessary for very high value of $H$. Considering the solution precision of moderately thick annular spherical shell, the value of $H$ is set as 6 in this paper.

The results of moderately thick annular spherical shell with different truncation numbers are shown in Figure 4. The example shows clearly that the current method converges quickly with the increase of $M$. To obtain the accurate solution of moderately thick annular spherical shell, the value of $M$ is defined as 8 in this study.

Figure 5 displays the results of moderately thick annular spherical shell with different Jacobi parameters $\alpha$ and $\beta$. The ordinate in this example is the relative percentage value, and the results of $\alpha=\beta=0.5$ are selected as the comparative object. It is apparently that the different Jacobi parameters have little effect on free vibration of moderately thick annular spherical shell. That is to say, all the Jacobi polynomials are capable to construct the displacement functions, and it 


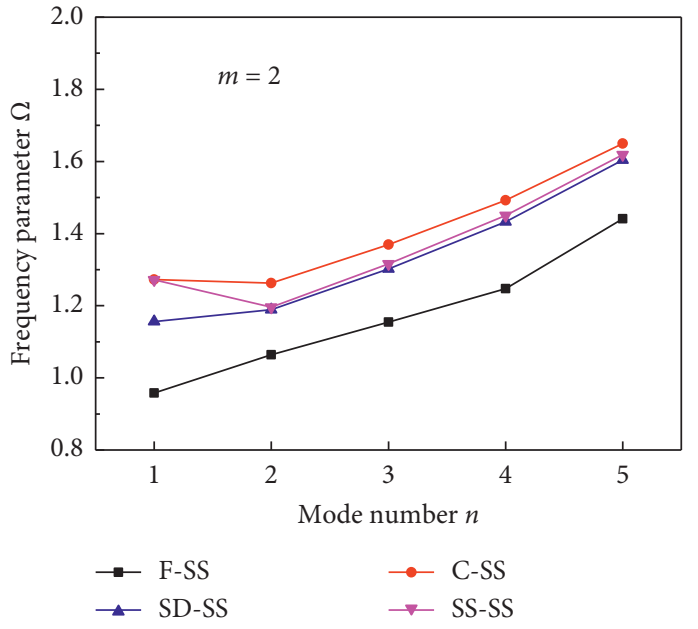

(a)

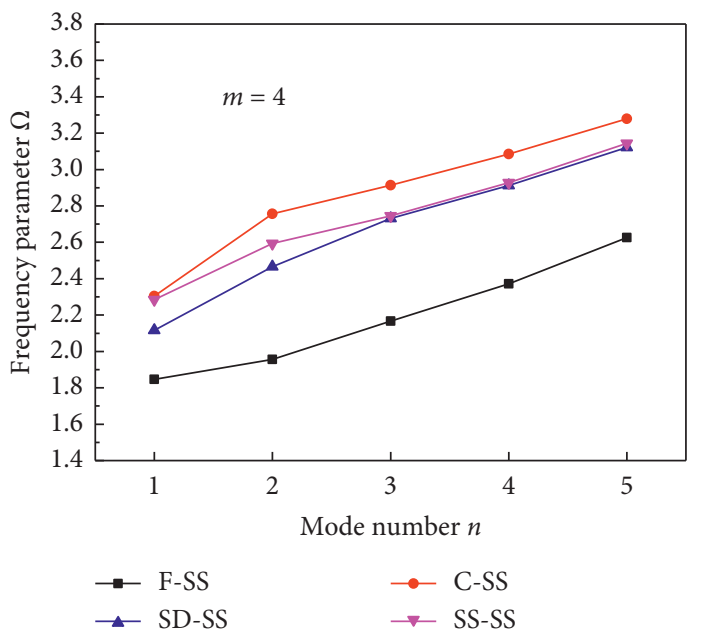

(c)

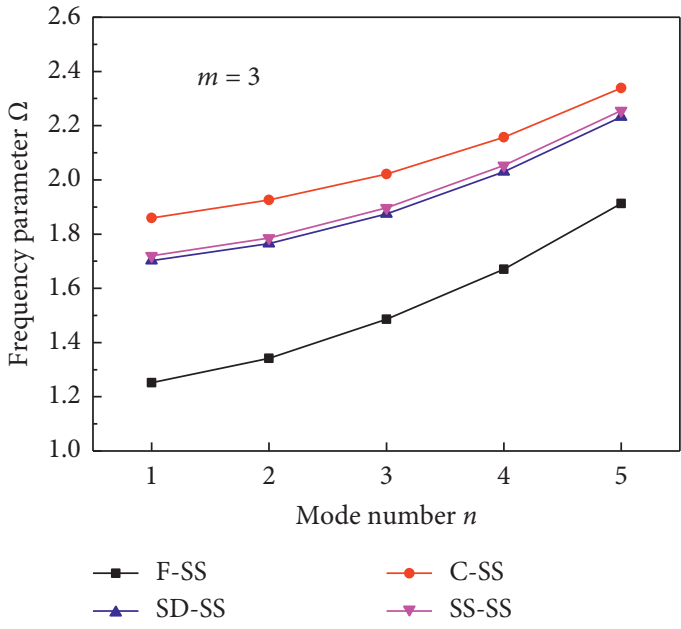

(b)

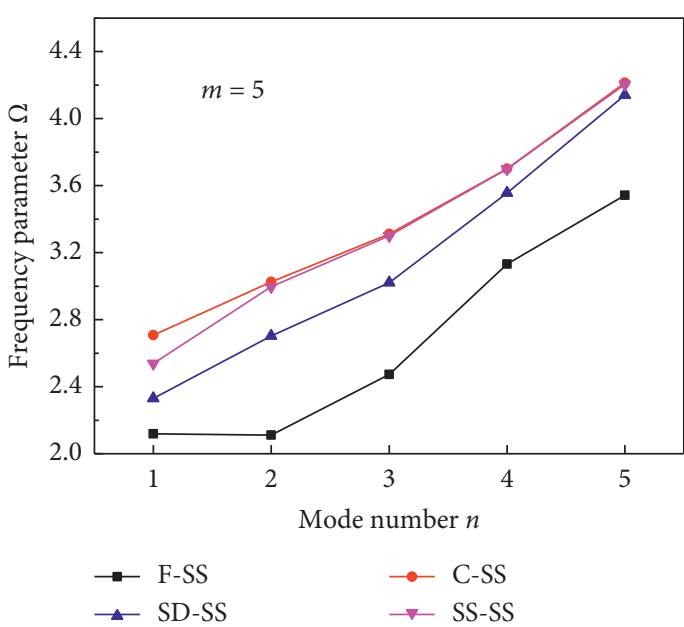

(d)

FIGURE 6: Frequency parameters $\Omega$ of the annular spherical shell with different boundary conditions.

can be regarded as one of the advantages of the current method.

3.2. Free Vibration Behavior of Moderately Thick Annular Spherical Shell Structure. Table 2 shows the natural frequency of moderately thick annular spherical shell with classical boundary conditions, and all the results are compared with those obtained in commercial FEM software ABAQUS. The geometrical parameters of model is defined as $\varphi_{0}=\pi / 6, \varphi_{1}=\pi / 2$, and $h=0.06 \mathrm{~m}$, and the meshes size are set as $0.01 \mathrm{~m}$. Table 3 exhibits the results of moderately thick annular spherical shell with classical boundary conditions, and the results are defined as $\Omega=\omega R \sqrt{\rho\left(1-\mu^{2}\right) / E}$. The geometrical parameters are defined as $\varphi_{0}=\pi / 8, \varphi_{1}=\pi / 2, h / R=0.05$, and $R=1 \mathrm{~m}$, and all the results are compared with Qu et al. [37]. From the above research, it is clear that the proposed method is very accurate in solving the free vibration of moderately thick annular spherical shell.

Tables 4 and 5 show the results of moderately thick annular spherical shell, respectively, which subject to classical and elastic edge restraints. Figure 6 displays the frequency parameters $\Omega$ under different boundary conditions which varies with the increasing of mode of $n$. From the examples, it is obviously that the different boundary conditions have a clear effect on the results of moderately thick annular spherical shell. To improve the understanding on free vibration characteristics of moderately thick annular spherical shell, some mode shapes under different boundary conditions are given in Figure 7.

Tables 6 and 7 and Figure 8 display the results with different $h / R$ ratios in moderately thick annular spherical shell. From the examples, it is obvious that that the results have a tendency to increase with $h / R$ increasing. Not surprisingly, the frequency parameters $\Omega$ increased obviously 


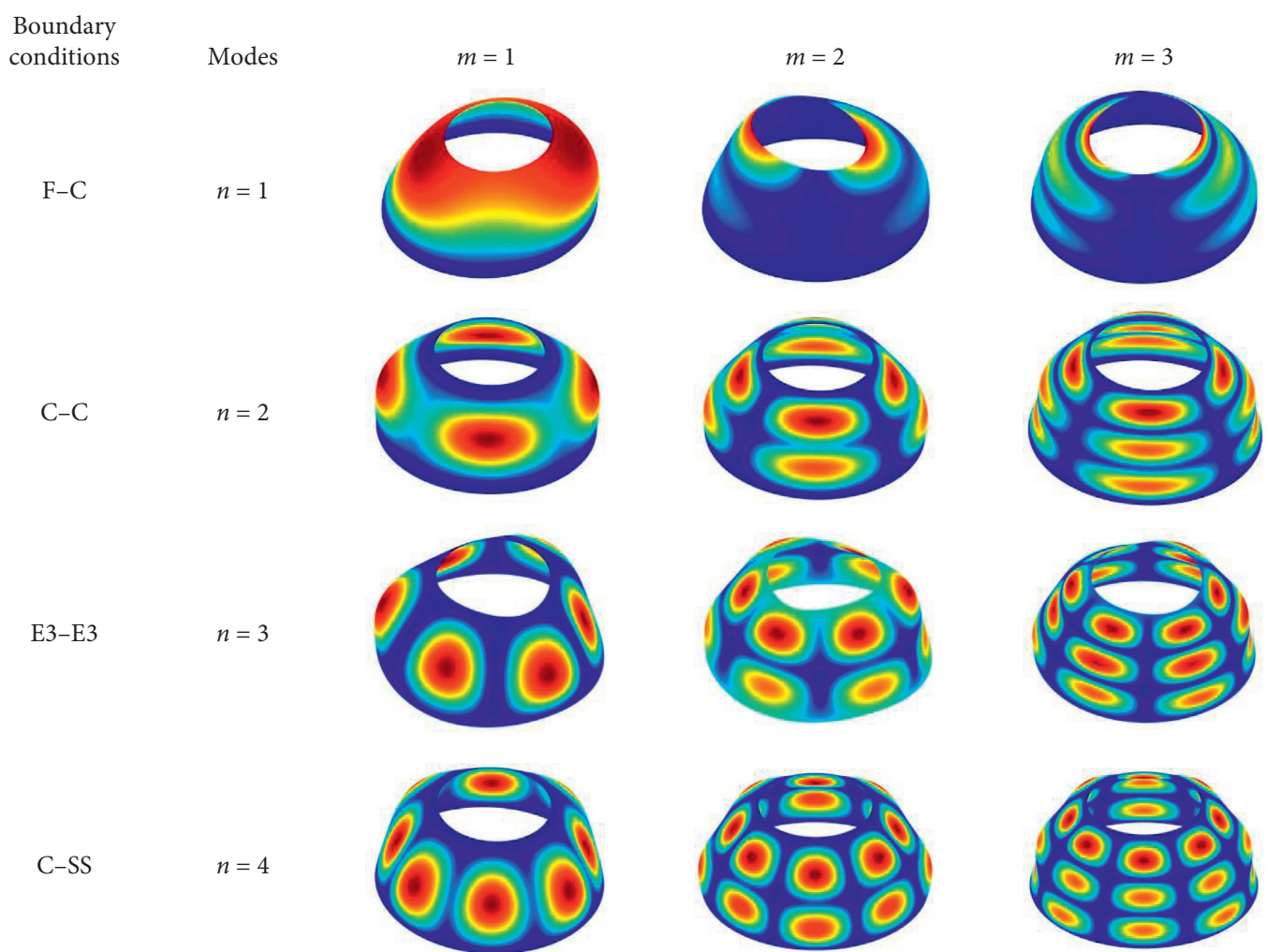

Figure 7: Comparison of the FEM and predicted mode shapes for the annular spherical shell structure.

TABle 6: Results of annular spherical shell with different $h / R$ ratios $(\varphi 0=\pi / 6, \varphi 1=\pi / 2, R=1 \mathrm{~m}$, and $n=1)$.

\begin{tabular}{|c|c|c|c|c|c|c|}
\hline \multirow{2}{*}{ Boundary conditions } & \multirow{2}{*}{$h / R$} & \multicolumn{5}{|c|}{$m$} \\
\hline & & 1 & 2 & 3 & 4 & 5 \\
\hline \multirow{5}{*}{$\mathrm{F}-\mathrm{C}$} & 0.04 & 0.6692 & 0.9562 & 1.1605 & 1.5797 & 2.0162 \\
\hline & 0.05 & 0.6756 & 0.9718 & 1.2491 & 1.7727 & 2.0984 \\
\hline & 0.06 & 0.6817 & 0.9894 & 1.3427 & 1.9047 & 2.1354 \\
\hline & 0.07 & 0.6877 & 1.0090 & 1.4362 & 1.9751 & 2.1365 \\
\hline & 0.08 & 0.6934 & 1.0304 & 1.5255 & 2.0214 & 2.1366 \\
\hline \multirow{5}{*}{ F-SS } & 0.04 & 0.6462 & 0.9349 & 1.1077 & 1.4789 & 2.0101 \\
\hline & 0.05 & 0.6484 & 0.9456 & 1.1760 & 1.6710 & 2.0767 \\
\hline & 0.06 & 0.6506 & 0.9577 & 1.2516 & 1.8469 & 2.1184 \\
\hline & 0.07 & 0.6528 & 0.9711 & 1.3316 & 1.9666 & 2.1361 \\
\hline & 0.08 & 0.6549 & 0.9858 & 1.4133 & 2.0203 & 2.1365 \\
\hline \multirow{5}{*}{$\mathrm{C}-\mathrm{C}$} & 0.04 & 1.1064 & 1.2307 & 1.6367 & 2.1884 & 2.3118 \\
\hline & 0.05 & 1.1903 & 1.2545 & 1.8287 & 2.2869 & 2.5964 \\
\hline & 0.06 & 1.2726 & 1.2795 & 2.0030 & 2.3335 & 2.8789 \\
\hline & 0.07 & 1.2893 & 1.3702 & 2.1229 & 2.4235 & 3.0382 \\
\hline & 0.08 & 1.3057 & 1.4598 & 2.1809 & 2.5630 & 3.1001 \\
\hline \multirow{5}{*}{ C-SS } & 0.04 & 1.0387 & 1.2263 & 1.5302 & 2.0551 & 2.2855 \\
\hline & 0.05 & 1.0977 & 1.2542 & 1.6909 & 2.2863 & 2.4067 \\
\hline & 0.06 & 1.1611 & 1.2727 & 1.8598 & 2.3046 & 2.7077 \\
\hline & 0.07 & 1.2224 & 1.2931 & 2.0162 & 2.3314 & 2.9422 \\
\hline & 0.08 & 1.2646 & 1.3327 & 2.1343 & 2.3903 & 3.0668 \\
\hline \multirow{5}{*}{ C-SD } & 0.04 & 0.7134 & 1.0543 & 1.3970 & 1.5920 & 2.0820 \\
\hline & 0.05 & 0.7228 & 1.1071 & 1.4891 & 1.6985 & 2.4045 \\
\hline & 0.06 & 0.7320 & 1.1643 & 1.5264 & 1.8602 & 2.5767 \\
\hline & 0.07 & 0.7411 & 1.2221 & 1.5500 & 2.0314 & 2.6179 \\
\hline & 0.08 & 0.7504 & 1.2770 & 1.5738 & 2.1938 & 2.6435 \\
\hline
\end{tabular}


TABle 6: Continued.

\begin{tabular}{|c|c|c|c|c|c|c|}
\hline \multirow{2}{*}{ Boundary conditions } & \multirow{2}{*}{$h / R$} & \multicolumn{5}{|c|}{$m$} \\
\hline & & 1 & 2 & 3 & 4 & 5 \\
\hline \multirow{5}{*}{$E 1-E 1$} & 0.04 & 0.6581 & 1.0742 & 1.2416 & 1.6043 & 2.1274 \\
\hline & 0.05 & 0.6625 & 1.1151 & 1.2956 & 1.8195 & 2.1364 \\
\hline & 0.06 & 0.6682 & 1.1437 & 1.3670 & 2.0209 & 2.1526 \\
\hline & 0.07 & 0.6747 & 1.1640 & 1.4497 & 2.1103 & 2.2708 \\
\hline & 0.08 & 0.6817 & 1.1804 & 1.5370 & 2.1204 & 2.4550 \\
\hline \multirow{5}{*}{$E 2-E 2$} & 0.04 & 0.6562 & 1.1071 & 1.4817 & 1.9230 & 2.1696 \\
\hline & 0.05 & 0.6647 & 1.1891 & 1.6149 & 2.0143 & 2.2345 \\
\hline & 0.06 & 0.6744 & 1.2766 & 1.6952 & 2.1306 & 2.2726 \\
\hline & 0.07 & 0.6849 & 1.3656 & 1.7404 & 2.1930 & 2.3867 \\
\hline & 0.08 & 0.6960 & 1.4532 & 1.7699 & 2.2105 & 2.5491 \\
\hline \multirow{5}{*}{$E 3-E 3$} & 0.04 & 0.2181 & 0.4215 & 1.1221 & 1.4347 & 1.6467 \\
\hline & 0.05 & 0.2007 & 0.4388 & 1.2062 & 1.4757 & 1.8292 \\
\hline & 0.06 & 0.1894 & 0.4576 & 1.2948 & 1.4920 & 2.0342 \\
\hline & 0.07 & 0.1820 & 0.4774 & 1.3808 & 1.5074 & 2.2341 \\
\hline & 0.08 & 0.1775 & 0.4978 & 1.4480 & 1.5397 & 2.4198 \\
\hline
\end{tabular}

TABLE 7: Results of annular spherical shell with different $h / R$ ratios $\left(\varphi_{0}=\pi / 6, \varphi_{1}=\pi / 2, R=1 \mathrm{~m}\right.$, and $\left.n=2\right)$.

\begin{tabular}{|c|c|c|c|c|c|c|}
\hline \multirow{2}{*}{ Boundary conditions } & \multirow{2}{*}{$h / R$} & \multicolumn{5}{|c|}{$m$} \\
\hline & & 1 & 2 & 3 & 4 & 5 \\
\hline \multirow{5}{*}{$\mathrm{F}-\mathrm{C}$} & 0.04 & 0.3173 & 1.0318 & 1.2223 & 1.6683 & 2.0346 \\
\hline & 0.05 & 0.3388 & 1.0646 & 1.3318 & 1.8857 & 2.0902 \\
\hline & 0.06 & 0.3605 & 1.1020 & 1.4457 & 1.9971 & 2.2387 \\
\hline & 0.07 & 0.3822 & 1.1431 & 1.5581 & 2.0342 & 2.4495 \\
\hline & 0.08 & 0.4039 & 1.1867 & 1.6631 & 2.0619 & 2.6536 \\
\hline \multirow{5}{*}{ F-SS } & 0.04 & 0.3000 & 1.0164 & 1.1589 & 1.5550 & 2.0328 \\
\hline & 0.05 & 0.3191 & 1.0385 & 1.2469 & 1.7688 & 2.0668 \\
\hline & 0.06 & 0.3382 & 1.0643 & 1.3421 & 1.9560 & 2.1109 \\
\hline & 0.07 & 0.3571 & 1.0932 & 1.4406 & 2.0338 & 2.2578 \\
\hline & 0.08 & 0.3756 & 1.1249 & 1.5391 & 2.0558 & 2.4519 \\
\hline \multirow{5}{*}{$\mathrm{C}-\mathrm{C}$} & 0.04 & 1.1066 & 1.1798 & 1.6578 & 2.2737 & 2.9366 \\
\hline & 0.05 & 1.1257 & 1.2714 & 1.8821 & 2.6280 & 2.9990 \\
\hline & 0.06 & 1.1451 & 1.3688 & 2.1017 & 2.8395 & 3.1365 \\
\hline & 0.07 & 1.1657 & 1.4681 & 2.3079 & 2.9045 & 3.2323 \\
\hline & 0.08 & 1.1874 & 1.5664 & 2.4943 & 2.9362 & 3.2494 \\
\hline \multirow{5}{*}{ C-SS } & 0.04 & 1.1007 & 1.1217 & 1.5389 & 2.1120 & 2.9141 \\
\hline & 0.05 & 1.1163 & 1.1881 & 1.7305 & 2.4621 & 2.9791 \\
\hline & 0.06 & 1.1290 & 1.2626 & 1.9256 & 2.7567 & 3.0247 \\
\hline & 0.07 & 1.1414 & 1.3413 & 2.1162 & 2.8988 & 3.1550 \\
\hline & 0.08 & 1.1543 & 1.4217 & 2.2976 & 2.9341 & 3.2243 \\
\hline \multirow{5}{*}{ C-SD } & 0.04 & 0.9349 & 1.1176 & 1.4644 & 1.6288 & 2.1378 \\
\hline & 0.05 & 0.9437 & 1.1841 & 1.5576 & 1.7428 & 2.4991 \\
\hline & 0.06 & 0.9540 & 1.2556 & 1.5831 & 1.9251 & 2.8368 \\
\hline & 0.07 & 0.9657 & 1.3281 & 1.5982 & 2.1151 & 3.1190 \\
\hline & 0.08 & 0.9785 & 1.3965 & 1.6159 & 2.2991 & 3.2221 \\
\hline \multirow{5}{*}{$E 1-E 1$} & 0.04 & 0.9466 & 1.1784 & 1.3930 & 1.6351 & 2.3054 \\
\hline & 0.05 & 0.9606 & 1.2694 & 1.3990 & 1.8645 & 2.6915 \\
\hline & 0.06 & 0.9770 & 1.3532 & 1.4163 & 2.0912 & 2.7441 \\
\hline & 0.07 & 0.9953 & 1.3832 & 1.4898 & 2.3070 & 2.7448 \\
\hline & 0.08 & 1.0152 & 1.3891 & 1.5870 & 2.5076 & 2.7457 \\
\hline \multirow{5}{*}{$E 2-E 2$} & 0.04 & 0.8823 & 1.1596 & 1.5964 & 2.2714 & 2.3228 \\
\hline & 0.05 & 0.9000 & 1.2514 & 1.8179 & 2.3222 & 2.6258 \\
\hline & 0.06 & 0.9195 & 1.3495 & 2.0245 & 2.3292 & 2.7795 \\
\hline & 0.07 & 0.9405 & 1.4497 & 2.1927 & 2.3539 & 2.8304 \\
\hline & 0.08 & 0.9628 & 1.5490 & 2.2714 & 2.4375 & 2.8825 \\
\hline \multirow{5}{*}{$E 3-E 3$} & 0.04 & 0.5684 & 0.6661 & 1.1828 & 1.5763 & 2.2037 \\
\hline & 0.05 & 0.5944 & 0.6853 & 1.2625 & 1.8042 & 2.2456 \\
\hline & 0.06 & 0.6201 & 0.7010 & 1.3519 & 2.0211 & 2.2574 \\
\hline & 0.07 & 0.6455 & 0.7142 & 1.4462 & 2.1883 & 2.3034 \\
\hline & 0.08 & 0.6694 & 0.7268 & 1.5414 & 2.2310 & 2.4565 \\
\hline
\end{tabular}




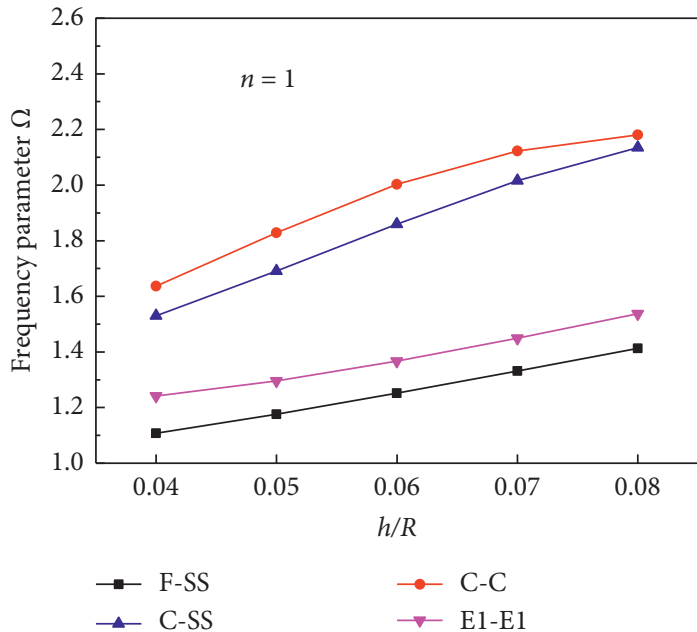

(a)

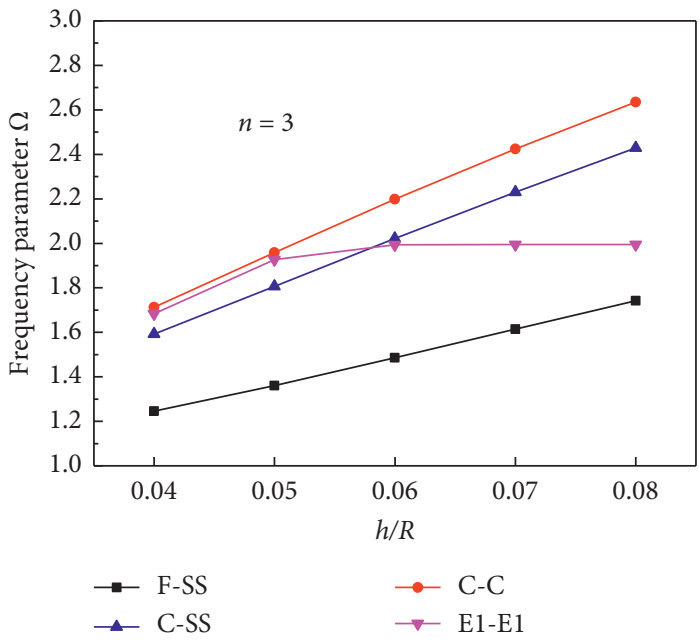

(c)

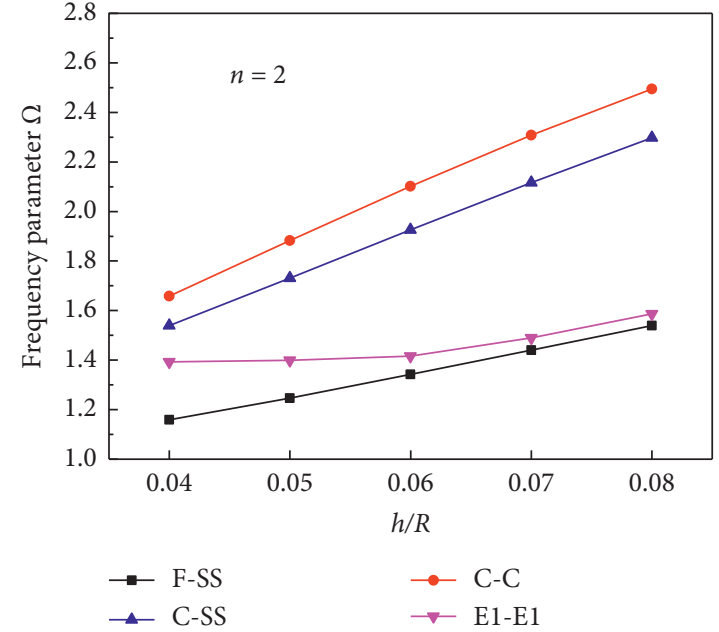

(b)

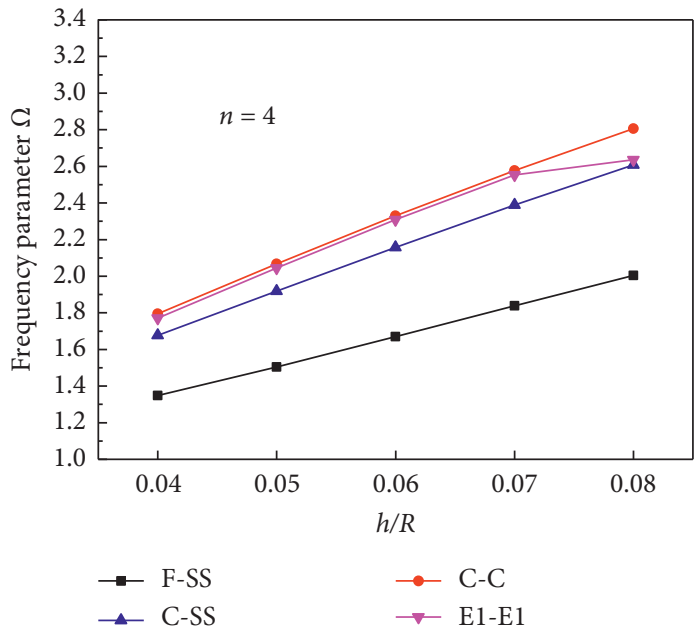

(d)

Figure 8: Frequency parameters $\Omega$ of the annular spherical shell with different $h / R$ ratios $(m=3)$.

TABLE 8: Results of annular spherical shell with different $\varphi_{0}\left(\varphi_{1}=\pi / 2, R=1 \mathrm{~m}, h=0.06 \mathrm{~m}\right.$, and $\left.n=1\right)$.

\begin{tabular}{|c|c|c|c|c|c|c|}
\hline \multirow{2}{*}{ Boundary conditions } & \multirow{2}{*}{$\varphi_{0}$} & \multicolumn{5}{|c|}{$m$} \\
\hline & & 1 & 2 & 3 & 4 & 5 \\
\hline \multirow{5}{*}{$\mathrm{F}-\mathrm{C}$} & $\pi / 8$ & 0.6436 & 0.9719 & 1.2359 & 1.7010 & 1.9829 \\
\hline & $\pi / 6$ & 0.6817 & 0.9894 & 1.3427 & 1.9047 & 2.1354 \\
\hline & $\pi / 5$ & 0.7207 & 1.0168 & 1.4858 & 2.0655 & 2.1588 \\
\hline & $\pi / 4$ & 0.7927 & 1.1003 & 1.8439 & 2.1205 & 2.4764 \\
\hline & $\pi / 3$ & 0.9324 & 1.6052 & 2.3732 & 3.2253 & 3.7807 \\
\hline \multirow{5}{*}{ F-SS } & $\pi / 8$ & 0.6151 & 0.9440 & 1.1715 & 1.6221 & 1.9529 \\
\hline & $\pi / 6$ & 0.6506 & 0.9577 & 1.2516 & 1.8469 & 2.1184 \\
\hline & $\pi / 5$ & 0.6869 & 0.9768 & 1.3646 & 2.0592 & 2.1445 \\
\hline & $\pi / 4$ & 0.7548 & 1.0298 & 1.6676 & 2.1047 & 2.4733 \\
\hline & $\pi / 3$ & 0.8847 & 1.3469 & 2.3711 & 3.0294 & 3.5040 \\
\hline \multirow{5}{*}{$\mathrm{C}-\mathrm{C}$} & $\pi / 8$ & 1.1348 & 1.1790 & 1.7292 & 2.1792 & 2.4499 \\
\hline & $\pi / 6$ & 1.2726 & 1.2795 & 2.0030 & 2.3335 & 2.8789 \\
\hline & $\pi / 5$ & 1.3572 & 1.4435 & 2.2659 & 2.5413 & 3.3283 \\
\hline & $\pi / 4$ & 1.5028 & 1.8395 & 2.6605 & 3.2375 & 4.1434 \\
\hline & $\pi / 3$ & 1.9826 & 3.4460 & 3.8057 & 6.0731 & 6.3115 \\
\hline
\end{tabular}


TABLE 8: Continued.

\begin{tabular}{|c|c|c|c|c|c|c|}
\hline \multirow{2}{*}{ Boundary conditions } & \multirow{2}{*}{$\varphi_{0}$} & \multicolumn{5}{|c|}{$m$} \\
\hline & & 1 & 2 & 3 & 4 & 5 \\
\hline \multirow{5}{*}{ C-SS } & $\pi / 8$ & 1.0521 & 1.1749 & 1.6077 & 2.1776 & 2.2916 \\
\hline & $\pi / 6$ & 1.1611 & 1.2727 & 1.8598 & 2.3046 & 2.7077 \\
\hline & $\pi / 5$ & 1.2836 & 1.3579 & 2.1425 & 2.4401 & 3.1824 \\
\hline & $\pi / 4$ & 1.4597 & 1.6187 & 2.6498 & 2.9327 & 4.0995 \\
\hline & $\pi / 3$ & 1.7692 & 2.9828 & 3.8054 & 5.5589 & 6.3115 \\
\hline \multirow{5}{*}{$\mathrm{C}-\mathrm{SD}$} & $\pi / 8$ & 0.6681 & 1.0615 & 1.3325 & 1.6075 & 2.2854 \\
\hline & $\pi / 6$ & 0.7320 & 1.1643 & 1.5264 & 1.8602 & 2.5767 \\
\hline & $\pi / 5$ & 0.7894 & 1.2834 & 1.7247 & 2.1488 & 2.7264 \\
\hline & $\pi / 4$ & 0.8963 & 1.5830 & 2.1195 & 2.7781 & 3.0742 \\
\hline & $\pi / 3$ & 1.2719 & 2.8503 & 3.3175 & 3.9809 & 5.5639 \\
\hline \multirow{5}{*}{$E 1-E 1$} & $\pi / 8$ & 0.6310 & 1.0695 & 1.2533 & 1.7324 & 2.1623 \\
\hline & $\pi / 6$ & 0.6682 & 1.1437 & 1.3670 & 2.0209 & 2.1526 \\
\hline & $\pi / 5$ & 0.6963 & 1.1792 & 1.5078 & 2.2043 & 2.3900 \\
\hline & $\pi / 4$ & 0.7349 & 1.2160 & 1.8837 & 2.5220 & 3.1666 \\
\hline & $\pi / 3$ & 0.7556 & 1.6250 & 3.4826 & 3.7092 & 6.0038 \\
\hline \multirow{5}{*}{$E 2-E 2$} & $\pi / 8$ & 0.6508 & 1.1457 & 1.5671 & 1.9046 & 2.1186 \\
\hline & $\pi / 6$ & 0.6744 & 1.2766 & 1.6952 & 2.1306 & 2.2726 \\
\hline & $\pi / 5$ & 0.6929 & 1.4366 & 1.7664 & 2.3607 & 2.4996 \\
\hline & $\pi / 4$ & 0.7327 & 1.7994 & 1.8725 & 2.7260 & 3.2327 \\
\hline & $\pi / 3$ & 0.8902 & 2.1320 & 3.4461 & 3.8783 & 6.0731 \\
\hline \multirow{5}{*}{$E 3-E 3$} & $\pi / 8$ & 0.1727 & 0.4252 & 1.1586 & 1.4577 & 1.7434 \\
\hline & $\pi / 6$ & 0.1894 & 0.4576 & 1.2948 & 1.4920 & 2.0342 \\
\hline & $\pi / 5$ & 0.2034 & 0.4968 & 1.4483 & 1.5153 & 2.3722 \\
\hline & $\pi / 4$ & 0.2269 & 0.5911 & 1.5123 & 1.8613 & 3.0009 \\
\hline & $\pi / 3$ & 0.2832 & 0.8905 & 1.7464 & 3.4707 & 4.0255 \\
\hline
\end{tabular}

TABLe 9: Results of annular spherical shell with different $\varphi_{0}\left(\varphi_{1}=\pi / 2, R=1 \mathrm{~m}, h=0.06 \mathrm{~m}\right.$, and $\left.n=2\right)$.

\begin{tabular}{|c|c|c|c|c|c|c|}
\hline \multirow{2}{*}{ Boundary conditions } & \multirow{2}{*}{$\varphi_{0}$} & \multicolumn{5}{|c|}{$m$} \\
\hline & & 1 & 2 & 3 & 4 & 5 \\
\hline \multirow{5}{*}{$\mathrm{F}-\mathrm{C}$} & $\pi / 8$ & 0.3989 & 1.0838 & 1.3492 & 1.8882 & 2.0273 \\
\hline & $\pi / 6$ & 0.3605 & 1.1020 & 1.4457 & 1.9971 & 2.2387 \\
\hline & $\pi / 5$ & 0.3825 & 1.1269 & 1.5822 & 2.1260 & 2.5498 \\
\hline & $\pi / 4$ & 0.4642 & 1.2032 & 1.9384 & 2.4367 & 2.9543 \\
\hline & $\pi / 3$ & 0.7003 & 1.6772 & 3.0515 & 3.2570 & 3.7890 \\
\hline \multirow{5}{*}{ F-SS } & $\pi / 8$ & 0.3866 & 1.0528 & 1.2677 & 1.7819 & 2.0039 \\
\hline & $\pi / 6$ & 0.3382 & 1.0643 & 1.3421 & 1.9560 & 2.1109 \\
\hline & $\pi / 5$ & 0.3516 & 1.0790 & 1.4502 & 2.1219 & 2.3433 \\
\hline & $\pi / 4$ & 0.4188 & 1.1248 & 1.7467 & 2.4282 & 2.9414 \\
\hline & $\pi / 3$ & 0.6185 & 1.4280 & 3.0188 & 3.1409 & 3.4721 \\
\hline \multirow{5}{*}{$\mathrm{C}-\mathrm{C}$} & $\pi / 8$ & 1.0663 & 1.2502 & 1.8101 & 2.4663 & 2.7955 \\
\hline & $\pi / 6$ & 1.1451 & 1.3688 & 2.1017 & 2.8395 & 3.1365 \\
\hline & $\pi / 5$ & 1.2226 & 1.5185 & 2.4320 & 3.0975 & 3.4107 \\
\hline & $\pi / 4$ & 1.3782 & 1.8981 & 3.1363 & 3.4427 & 4.0992 \\
\hline & $\pi / 3$ & 1.9276 & 3.4851 & 4.2397 & 6.1067 & 6.2292 \\
\hline \multirow{5}{*}{ C-SS } & $\pi / 8$ & 1.0554 & 1.1742 & 1.6726 & 2.3405 & 2.7515 \\
\hline & $\pi / 6$ & 1.1290 & 1.2626 & 1.9256 & 2.7567 & 3.0247 \\
\hline & $\pi / 5$ & 1.1987 & 1.3767 & 2.2190 & 3.0967 & 3.2830 \\
\hline & $\pi / 4$ & 1.3275 & 1.6774 & 2.9001 & 3.3744 & 4.0740 \\
\hline & $\pi / 3$ & 1.7085 & 3.0270 & 4.2397 & 5.5981 & 6.2292 \\
\hline \multirow{5}{*}{ C-SD } & $\pi / 8$ & 0.9343 & 1.1710 & 1.4259 & 1.6727 & 2.3894 \\
\hline & $\pi / 6$ & 0.9540 & 1.2556 & 1.5831 & 1.9251 & 2.8368 \\
\hline & $\pi / 5$ & 0.9749 & 1.3660 & 1.7503 & 2.2177 & 3.2817 \\
\hline & $\pi / 4$ & 1.0298 & 1.6562 & 2.0976 & 2.9028 & 3.6387 \\
\hline & $\pi / 3$ & 1.3407 & 2.8863 & 3.2496 & 4.5122 & 5.6032 \\
\hline
\end{tabular}


TABle 9: Continued.

\begin{tabular}{|c|c|c|c|c|c|c|}
\hline \multirow{2}{*}{ Boundary conditions } & \multirow{2}{*}{$\varphi_{0}$} & \multicolumn{5}{|c|}{$m$} \\
\hline & & 1 & 2 & 3 & 4 & 5 \\
\hline \multirow{5}{*}{$E 1-E 1$} & $\pi / 8$ & 0.9479 & 1.2473 & 1.3445 & 1.7968 & 2.5694 \\
\hline & $\pi / 6$ & 0.9770 & 1.3532 & 1.4163 & 2.0912 & 2.7441 \\
\hline & $\pi / 5$ & 1.0107 & 1.4143 & 1.5423 & 2.4289 & 2.7437 \\
\hline & $\pi / 4$ & 1.0983 & 1.4399 & 1.9199 & 2.9001 & 3.2094 \\
\hline & $\pi / 3$ & 1.3349 & 1.6564 & 3.5149 & 3.9016 & 6.0480 \\
\hline \multirow{5}{*}{$E 2-E 2$} & $\pi / 8$ & 0.8894 & 1.2273 & 1.7592 & 2.1700 & 2.5066 \\
\hline & $\pi / 6$ & 0.9195 & 1.3495 & 2.0245 & 2.3292 & 2.7795 \\
\hline & $\pi / 5$ & 0.9510 & 1.5023 & 2.2801 & 2.5156 & 2.8662 \\
\hline & $\pi / 4$ & 1.0284 & 1.8866 & 2.5227 & 2.9265 & 3.3212 \\
\hline & $\pi / 3$ & 1.4026 & 2.6416 & 3.4850 & 4.0183 & 6.1066 \\
\hline \multirow{5}{*}{$E 3-E 3$} & $\pi / 8$ & 0.6189 & 0.7555 & 1.2454 & 1.7337 & 2.1863 \\
\hline & $\pi / 6$ & 0.6201 & 0.7010 & 1.3519 & 2.0211 & 2.2574 \\
\hline & $\pi / 5$ & 0.6202 & 0.6865 & 1.4994 & 2.2344 & 2.4279 \\
\hline & $\pi / 4$ & 0.5772 & 0.7677 & 1.8830 & 2.3026 & 3.1813 \\
\hline & $\pi / 3$ & 0.4831 & 1.2823 & 2.3369 & 3.4941 & 4.5082 \\
\hline
\end{tabular}

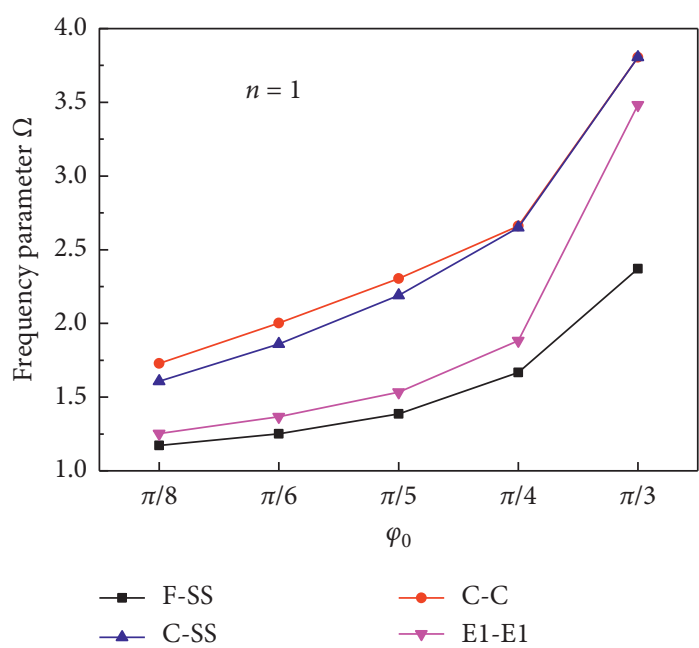

(a)

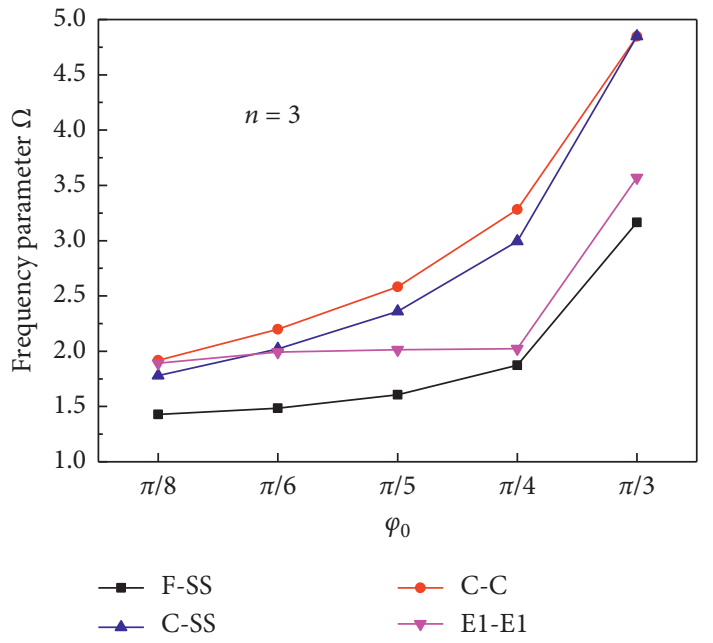

(c)

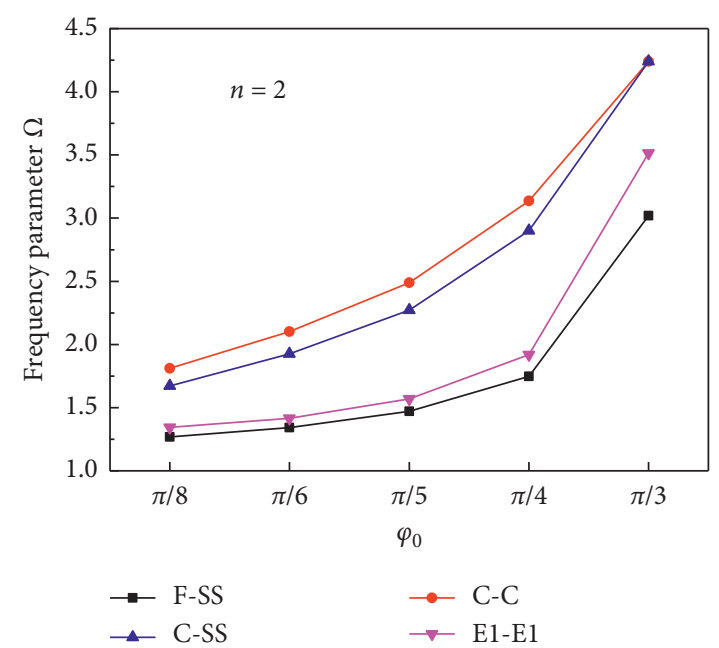

(b)

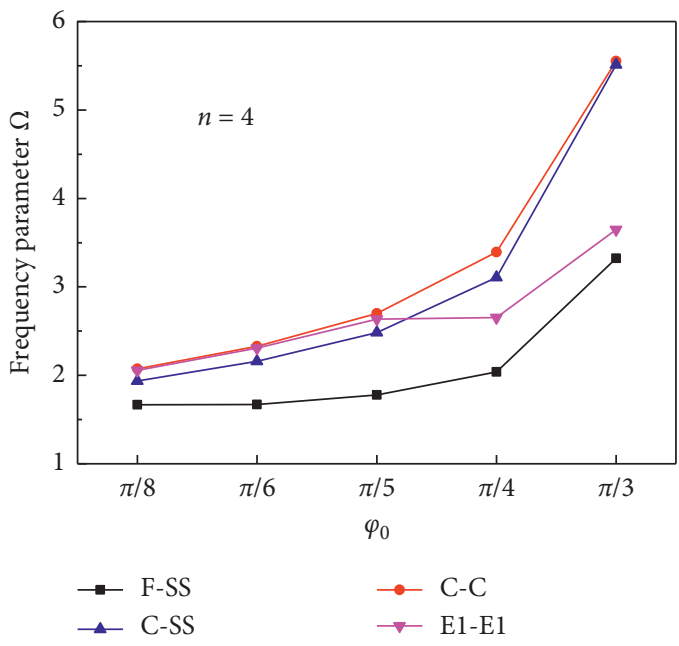

(d)

FIGURE 9: Frequency parameters $\Omega$ of the annular spherical shell with different $\varphi_{0}(m=3)$. 
TABLE 10: Results of annular spherical shell with different $\varphi_{1}\left(\varphi_{0}=\pi / 8, R=1 \mathrm{~m}, h=0.06 \mathrm{~m}\right.$, and $\left.n=1\right)$.

\begin{tabular}{|c|c|c|c|c|c|c|}
\hline \multirow{2}{*}{ Boundary conditions } & \multirow{2}{*}{$\Phi_{1}$} & \multicolumn{5}{|c|}{$m$} \\
\hline & & 1 & 2 & 3 & 4 & 5 \\
\hline \multirow{5}{*}{$\mathrm{F}-\mathrm{C}$} & $\pi / 6$ & 3.6431 & 8.9681 & 12.6428 & 13.2118 & 22.9000 \\
\hline & $\pi / 5$ & 1.6899 & 5.4835 & 5.8583 & 7.6132 & 12.5239 \\
\hline & $\pi / 4$ & 1.1818 & 2.6133 & 3.8455 & 4.9257 & 5.9473 \\
\hline & $\pi / 3$ & 0.9814 & 1.3664 & 2.4315 & 2.8442 & 3.3609 \\
\hline & $\pi / 2$ & 0.6436 & 0.9719 & 1.2359 & 1.7010 & 1.9829 \\
\hline \multirow{5}{*}{ F-SS } & $\pi / 6$ & 1.3547 & 8.9681 & 11.6078 & 13.0808 & 22.8995 \\
\hline & $\pi / 5$ & 1.1804 & 4.6180 & 5.6506 & 7.6055 & 11.5638 \\
\hline & $\pi / 4$ & 1.0825 & 2.0957 & 3.8404 & 4.8865 & 5.2709 \\
\hline & $\pi / 3$ & 0.9527 & 1.2149 & 2.2277 & 2.7311 & 3.3606 \\
\hline & $\pi / 2$ & 0.6152 & 0.9440 & 1.1715 & 1.6221 & 1.9529 \\
\hline \multirow{5}{*}{$\mathrm{C}-\mathrm{C}$} & $\pi / 6$ & 11.8237 & 15.0427 & 23.1127 & 25.1201 & 29.8383 \\
\hline & $\pi / 5$ & 5.4266 & 8.5302 & 11.2836 & 14.0476 & 16.7648 \\
\hline & $\pi / 4$ & 2.6824 & 5.2672 & 5.4583 & 8.4610 & 9.3175 \\
\hline & $\pi / 3$ & 1.6279 & 2.4319 & 3.3373 & 4.3047 & 5.0647 \\
\hline & $\pi / 2$ & 1.1348 & 1.1790 & 1.7292 & 2.1793 & 2.4499 \\
\hline \multirow{5}{*}{ C-SS } & $\pi / 6$ & 9.7987 & 15.0422 & 22.6127 & 25.1183 & 29.8370 \\
\hline & $\pi / 5$ & 4.2515 & 8.5296 & 10.3896 & 14.0384 & 16.7648 \\
\hline & $\pi / 4$ & 2.1955 & 4.7569 & 5.2871 & 8.4503 & 8.6874 \\
\hline & $\pi / 3$ & 1.5333 & 2.0992 & 3.3372 & 3.8881 & 5.0582 \\
\hline & $\pi / 2$ & 1.0521 & 1.1750 & 1.6077 & 2.1776 & 2.2916 \\
\hline \multirow{5}{*}{ C-SD } & $\pi / 6$ & 9.6128 & 12.3549 & 15.2373 & 22.6804 & 29.6584 \\
\hline & $\pi / 5$ & 3.9637 & 6.7642 & 8.8407 & 10.4648 & 16.5420 \\
\hline & $\pi / 4$ & 1.7120 & 3.9412 & 4.8167 & 5.7316 & 8.6811 \\
\hline & $\pi / 3$ & 0.8811 & 2.0460 & 2.4136 & 3.7170 & 3.9839 \\
\hline & $\pi / 2$ & 0.6681 & 1.0615 & 1.3325 & 1.6075 & 2.2854 \\
\hline \multirow{5}{*}{$E 1-E 1$} & $\pi / 6$ & 2.5277 & 11.7245 & 14.9480 & 23.1578 & 25.3798 \\
\hline & $\pi / 5$ & 2.1853 & 5.2760 & 8.4169 & 11.3354 & 14.3567 \\
\hline & $\pi / 4$ & 1.6668 & 2.5613 & 5.1988 & 5.4749 & 8.8397 \\
\hline & $\pi / 3$ & 0.9140 & 1.6802 & 2.4653 & 3.3383 & 4.2588 \\
\hline & $\pi / 2$ & 0.6310 & 1.0695 & 1.2533 & 1.7324 & 2.1624 \\
\hline \multirow{5}{*}{$E 2-E 2$} & $\pi / 6$ & 2.4058 & 11.8290 & 15.1668 & 23.1153 & 25.2815 \\
\hline & $\pi / 5$ & 2.0827 & 5.4594 & 8.6829 & 11.2863 & 14.3044 \\
\hline & $\pi / 4$ & 1.5810 & 2.8691 & 5.4120 & 5.4854 & 8.8295 \\
\hline & $\pi / 3$ & 0.9655 & 2.0415 & 2.4579 & 3.4721 & 4.3036 \\
\hline & $\pi / 2$ & 0.6508 & 1.1457 & 1.5671 & 1.9046 & 2.1187 \\
\hline \multirow{5}{*}{$E 3-E 3$} & $\pi / 6$ & 0.6065 & 3.0523 & 11.7244 & 15.3288 & 23.1578 \\
\hline & $\pi / 5$ & 0.4401 & 2.6234 & 5.2828 & 8.9518 & 11.3361 \\
\hline & $\pi / 4$ & 0.3274 & 1.8676 & 2.6981 & 5.4573 & 5.8831 \\
\hline & $\pi / 3$ & 0.2409 & 0.8808 & 1.9341 & 2.4549 & 4.0376 \\
\hline & $\pi / 2$ & 0.1727 & 0.4252 & 1.1586 & 1.4577 & 1.7434 \\
\hline
\end{tabular}

TABLe 11: Results of annular spherical shell with different $\varphi_{1}\left(\varphi_{0}=\pi / 2, R=1 \mathrm{~m}, h=0.06 \mathrm{~m}\right.$, and $\left.n=2\right)$.

\begin{tabular}{|c|c|c|c|c|c|c|}
\hline \multirow{2}{*}{ Boundary conditions } & \multirow{2}{*}{$\varphi_{1}$} & \multicolumn{5}{|c|}{$m$} \\
\hline & & 1 & 2 & 3 & 4 & 5 \\
\hline \multirow{5}{*}{$\mathrm{F}-\mathrm{C}$} & $\pi / 6$ & 3.8150 & 9.7744 & 12.7949 & 13.2675 & 23.3452 \\
\hline & $\pi / 5$ & 1.7976 & 5.7957 & 6.5679 & 7.9042 & 12.6403 \\
\hline & $\pi / 4$ & 1.0786 & 2.8407 & 4.3014 & 5.6835 & 6.0835 \\
\hline & $\pi / 3$ & 0.6610 & 1.5900 & 2.6462 & 3.0491 & 4.1950 \\
\hline & $\pi / 2$ & 0.3990 & 1.0838 & 1.3492 & 1.8882 & 2.0273 \\
\hline \multirow{5}{*}{ F-SS } & $\pi / 6$ & 1.7894 & 9.7735 & 11.8147 & 13.1186 & 23.3427 \\
\hline & $\pi / 5$ & 1.2525 & 4.8691 & 6.4732 & 7.9034 & 11.7076 \\
\hline & $\pi / 4$ & 0.8858 & 2.3609 & 4.3011 & 5.3930 & 5.6844 \\
\hline & $\pi / 3$ & 0.5942 & 1.4280 & 2.4131 & 2.9757 & 4.1595 \\
\hline & $\pi / 2$ & 0.3866 & 1.0528 & 1.2677 & 1.7819 & 2.0040 \\
\hline
\end{tabular}


TABLE 11: Continued.

\begin{tabular}{|c|c|c|c|c|c|c|}
\hline \multirow{2}{*}{ Boundary conditions } & \multirow{2}{*}{$\varphi_{1}$} & \multicolumn{5}{|c|}{$m$} \\
\hline & & 1 & 2 & 3 & 4 & 5 \\
\hline \multirow{5}{*}{$\mathrm{C}-\mathrm{C}$} & $\pi / 6$ & 11.8721 & 15.5416 & 23.1968 & 25.0071 & 30.2760 \\
\hline & $\pi / 5$ & 5.4699 & 9.2255 & 11.3802 & 13.9153 & 17.3881 \\
\hline & $\pi / 4$ & 2.6771 & 5.5250 & 6.1150 & 8.3655 & 9.4051 \\
\hline & $\pi / 3$ & 1.5265 & 2.5309 & 4.0903 & 4.4366 & 5.0917 \\
\hline & $\pi / 2$ & 1.0663 & 1.2502 & 1.8101 & 2.4664 & 2.7956 \\
\hline \multirow{5}{*}{ C-SS } & $\pi / 6$ & 9.9024 & 15.5405 & 22.6995 & 25.0063 & 30.2709 \\
\hline & $\pi / 5$ & 4.3291 & 9.2250 & 10.4991 & 13.9064 & 17.3881 \\
\hline & $\pi / 4$ & 2.1852 & 4.8709 & 6.1028 & 8.3585 & 8.7829 \\
\hline & $\pi / 3$ & 1.4057 & 2.2061 & 3.9147 & 4.2136 & 5.0848 \\
\hline & $\pi / 2$ & 1.0555 & 1.1742 & 1.6726 & 2.3405 & 2.7516 \\
\hline \multirow{5}{*}{$\mathrm{C}-\mathrm{SD}$} & $\pi / 6$ & 9.7048 & 12.0543 & 16.1126 & 22.7599 & 29.7101 \\
\hline & $\pi / 5$ & 4.0875 & 6.4610 & 9.9060 & 10.5863 & 16.6106 \\
\hline & $\pi / 4$ & 1.8688 & 3.7454 & 4.9063 & 6.8564 & 8.7765 \\
\hline & $\pi / 3$ & 1.0948 & 2.1426 & 2.3305 & 3.9390 & 4.8355 \\
\hline & $\pi / 2$ & 0.9344 & 1.1710 & 1.4259 & 1.6727 & 2.3895 \\
\hline \multirow{5}{*}{$E 1-E 1$} & $\pi / 6$ & 3.4567 & 11.7824 & 15.1735 & 23.2436 & 25.6980 \\
\hline & $\pi / 5$ & 2.9763 & 5.3506 & 8.8059 & 11.4264 & 14.7994 \\
\hline & $\pi / 4$ & 2.2170 & 2.6997 & 5.5649 & 5.8007 & 9.2446 \\
\hline & $\pi / 3$ & 1.2500 & 1.8752 & 2.5440 & 4.0978 & 4.3435 \\
\hline & $\pi / 2$ & 0.9479 & 1.2473 & 1.3445 & 1.7968 & 2.5694 \\
\hline \multirow{5}{*}{$E 2-E 2$} & $\pi / 6$ & 4.6941 & 11.8925 & 15.3560 & 23.2065 & 25.6246 \\
\hline & $\pi / 5$ & 3.9936 & 5.6479 & 8.9445 & 11.3881 & 14.8358 \\
\hline & $\pi / 4$ & 2.3491 & 3.8445 & 5.5347 & 5.7751 & 9.4039 \\
\hline & $\pi / 3$ & 1.2645 & 2.4977 & 3.0776 & 3.7952 & 4.4128 \\
\hline & $\pi / 2$ & 0.8894 & 1.2273 & 1.7592 & 2.1700 & 2.5066 \\
\hline \multirow{5}{*}{$E 3-E 3$} & $\pi / 6$ & 0.7960 & 4.8777 & 11.7827 & 15.9717 & 23.2436 \\
\hline & $\pi / 5$ & 0.8284 & 4.1400 & 5.4124 & 9.8944 & 11.4306 \\
\hline & $\pi / 4$ & 0.8871 & 2.2813 & 3.6241 & 5.5559 & 7.0605 \\
\hline & $\pi / 3$ & 0.8699 & 1.0742 & 2.4766 & 2.8647 & 4.2797 \\
\hline & $\pi / 2$ & 0.6189 & 0.7555 & 1.2455 & 1.7337 & 2.1863 \\
\hline
\end{tabular}

with the increase of mode of $m$. Tables 8 and 9 and Figure 9 exhibit the results with different $\varphi_{0}$ in moderately thick annular spherical shell. In addition, the results with different $\varphi_{1}$ in moderately thick annular spherical shell are shown in Tables 10 and 11 , in which $\varphi_{0}=\pi / 8$. From the examples, it is remarkable that the frequency parameters $\Omega$ tend to increase with the increase of $\varphi_{0}$, and the results usually decrease monotonously as the $\varphi_{1}$ increases, that is to say higher frequency parameters can be obtained as the structural scale decreases. Beyond that we can also see that the different boundary conditions have apparent effects on the frequency parameters $\Omega$.

\section{Conclusions}

This paper introduced a unified computational Ritz method to investigate free vibration characteristics of isotropic moderately thick annular spherical shell with general boundary conditions. The energy expressions of the annular spherical shell were established based on FSDT. The spring stiffness method is introduced to guarantee continuity and simulate various boundary conditions on the basic of the domain decomposition method. Beyond that the unified
Jacobi polynomials and Fourier series are, respectively, applied to represent the displacement admissible function along axial direction and circumferential direction of the structure. The final solutions can be obtained according to the Ritz method. In order to prove the validity of the proposed method, the results of the same condition are compared with those obtained by the finite element method and published literatures. The results show that the current method has fast convergence and delightful accuracy through the comparative study. The most discovery of the current method is that the selection of admissible displacement functions is generalized by applying Jacobi polynomial. In addition, the effects on the boundary conditions, shell segments, and structural parameters are presented through a large number of numerical examples, which can be used as basic data to provide reference for future staff of such research in this field.

\section{Data Availability}

The data used to support the findings of this study are included within the article. 


\section{Conflicts of Interest}

The authors declare that there are no conflicts of interest regarding the publication of this paper.

\section{Acknowledgments}

This study was funded by National Key Research and Development Program (2016YFC0303406), Key Research and Development Program of Shandong Province (2019JZZY010125), National Key Laboratory on Ship Vibration \& Noise Fund of China (6142204190207), National Natural Science Foundation of China (51709063), and Ph.D. Student Research and Innovation Fund of the Fundamental Research Funds for the Central Universities (HEUGIP201902).

\section{References}

[1] A. Bryan, "Free vibration of thin spherical shells," Journal of Vibration and Acoustics-Transactions of the ASME, vol. 139, no. 6, 2017.

[2] K. Xie, M. Chen, W. Dong, and W. Li, "A unified semi-analytical method for vibration analysis of shells of revolution stiffened by rings with T cross-section," Thin-Walled Structures, vol. 139, pp. 412-431, 2019.

[3] Q. Wang, K. Choe, D. Shi, and K. Sin, "Vibration analysis of the coupled doubly-curved revolution shell structures by using Jacobi-Ritz method," International Journal of $\mathrm{Me}$ chanical Sciences, vol. 135, pp. 517-531, 2018.

[4] F. Pang, H. Li, X. Wang, X. Miao, and S. Li, "A semi analytical method for the free vibration of doubly-curved shells of revolution," Computers \& Mathematics with Applications, vol. 75, no. 9, pp. 3249-3268, 2018.

[5] S. Li and H. Yuan, "Green quasifunction method for free vibration of simply-supported trapezoidal shallow spherical shell on Winkler foundation," Acta Mechanica Solida Sinica, vol. 23, no. 4, pp. 370-376, 2010.

[6] S.-Q. Li and H. Yuan, "Quasi-Green's function method for free vibration of simply-supported trapezoidal shallow spherical shell," Applied Mathematics and Mechanics, vol. 31, no. 5, pp. 635-642, 2010.

[7] M. Menaa and A. A. Lakis, "Free vibration of spherical shells using a hybrid finite element method," International Journal of Structural Stability and Dynamics, vol. 15, no. 4, 2015.

[8] R. Zaera, J. Fernández-Sáez, and J. A. Loya, "Axisymmetric free vibration of closed thin spherical nano-shell," Composite Structures, vol. 104, pp. 154-161, 2013.

[9] V. A. Polyakov, R. P. Shlitsa, V. V. Khitrov, and V. I. Zhigun, "An applied model for free radial vibrations of a closed spherical sandwich shell," Mechanics of Composite Materials, vol. 43, no. 4, pp. 331-344, 2007.

[10] O. Thomas, C. Touze, and A. Chaigne, "Non-linear vibrations of free-edge thin spherical shells: modal interaction rules and 1: 1: 2 internal resonance," International Journal of Solids and Structures, vol. 42, no. 11-12, pp. 3339-3373, 2005.

[11] O. Thomas, C. Touze, and E. Luminais, "Non-linear vibrations of free-edge thin spherical shells: experiments on a $1: 1: 2$ internal resonance," Nonlinear Dynamics, vol. 49, no. 1-2, pp. 259-284, 2007.

[12] F. Tornabene, S. Brischetto, N. Fantuzzi, and E. Viola, "Numerical and exact models for free vibration analysis of cylindrical and spherical shell panels," Composites Part B: Engineering, vol. 81, pp. 231-250, 2015.
[13] F. Tomabene, N. Fantuzzi, and M. Bacciocchi, "Free vibrations of free-form doubly-curved shells made of functionally graded materials using higher-order equivalent single layer theories," Composites Part B-Engineering, vol. 67, pp. 490-509, 2014.

[14] F. Tornabene and E. Viola, "Free vibrations of four-parameter functionally graded parabolic panels and shells of revolution," European Journal of Mechanics-A/Solids, vol. 28, no. 5, pp. 991-1013, 2009.

[15] F. Tornabene, "2-D GDQ solution for free vibrations of anisotropic doubly-curved shells and panels of revolution," Composite Structures, vol. 93, no. 7, pp. 1854-1876, 2011.

[16] F. Tornabene and S. Brischetto, "3D capability of refined GDQ models for the bending analysis of composite and sandwich plates, spherical and doubly-curved shells," Thin-Walled Structures, vol. 129, pp. 94-124, 2018.

[17] G. R. Buchanan and B. S. Rich, "Effect of boundary conditions on free vibration of thick isotropic spherical shells," Journal of Vibration and Control, vol. 8, no. 3, pp. 389-403, 2002.

[18] J. Guo, D. Shi, Q. Wang, J. Tang, and C. Shuai, "Dynamic analysis of laminated doubly-curved shells with general boundary conditions by means of a domain decomposition method," International Journal of Mechanical Sciences, vol. 138-139, pp. 159-186, 2018.

[19] Q. Wang, F. Pang, B. Qin, and Q. Liang, “A unified formulation for free vibration of functionally graded carbon nanotube reinforced composite spherical panels and shells of revolution with general elastic restraints by means of the Rayleigh-Ritz method," Polymer Composites, vol. 39, no. S2, pp. E924-E944, 2018.

[20] Q. Wang, D. Shi, Q. Liang, and F. Pang, "Free vibration of four-parameter functionally graded moderately thick doublycurved panels and shells of revolution with general boundary conditions," Applied Mathematical Modelling, vol. 42, pp. 705-734, 2017.

[21] Q. Wang, X. Cui, B. Qin, Q. Liang, and J. Tang, "A semianalytical method for vibration analysis of functionally graded (FG) sandwich doubly-curved panels and shells of revolution," International Journal of Mechanical Sciences, vol. 134, pp. 479-499, 2017.

[22] X. Xie, H. Zheng, and G. Jin, "Free vibration of four-parameter functionally graded spherical and parabolic shells of revolution with arbitrary boundary conditions," Composites Part B: Engineering, vol. 77, pp. 59-73, 2015.

[23] T. Ye, G. Jin, and Z. Su, "Three-dimensional vibration analysis of laminated functionally graded spherical shells with general boundary conditions," Composite Structures, vol. 116, pp. 571-588, 2014.

[24] T. Ye, G. Jin, and Z. Su, "Three-dimensional vibration analysis of functionally graded sandwich deep open spherical and cylindrical shells with general restraints," Journal of Vibration and Control, vol. 22, no. 15, pp. 3326-3354, 2016.

[25] Z. Su, G. Jin, S. Shi, and T. Ye, "A unified accurate solution for vibration analysis of arbitrary functionally graded spherical shell segments with general end restraints," Composite Structures, vol. 111, pp. 271-284, 2014.

[26] S. Hosseini-Hashemi and M. Fadaee, "On the free vibration of moderately thick spherical shell panel-a new exact closedform procedure," Journal of Sound and Vibration, vol. 330, no. 17, pp. 4352-4367, 2011.

[27] A. M. Zenkour, "Global structural behaviour of thin and moderately thick monoclinic spherical shells using a mixed shear deformation model," Archive of Applied Mechanics, vol. 74, no. 3-4, pp. 262-276, 2004. 
[28] F. A. Fazzolari, "A refined dynamic stiffness element for free vibration analysis of cross-ply laminated composite cylindrical and spherical shallow shells," Composites Part B: Engineering, vol. 62, pp. 143-158, 2014.

[29] S. K. Panda and B. N. Singh, "Nonlinear free vibration of spherical shell panel using higher order shear deformation theory-a finite element approach," International Journal of Pressure Vessels and Piping, vol. 86, no. 6, pp. 373-383, 2009.

[30] S. K. Panda and B. N. Singh, "Nonlinear free vibration analysis of thermally post-buckled composite spherical shell panel," International Journal of Mechanics and Materials in Design, vol. 6, no. 2, pp. 175-188, 2010.

[31] V. K. Singh and S. K. Panda, "Linear static and free vibration analyses of laminated composite spherical shells," Proceedings of the ASME Gas Turbine India Conference, p. 2014, 2013.

[32] V. K. Singh and S. K. Panda, "Large amplitude free vibration analysis of laminated composite spherical shells embedded with piezoelectric layers," Smart Structures and Systems, vol. 16, no. 5, pp. 853-872, 2015.

[33] H. Li, F. Pang, and H. Chen, "A semi-analytical approach to analyze vibration characteristics of uniform and stepped annular-spherical shells with general boundary conditions," European Journal of Mechanics-A/Solids, vol. 74, pp. 48-65, 2019.

[34] F. Pang, H. Li, J. Cui, Y. Du, and C. Gao, "Application of flügge thin shell theory to the solution of free vibration behaviors for spherical-cylindrical-spherical shell: a unified formulation," European Journal of Mechanics-A/Solids, vol. 74, pp. 381-393, 2019.

[35] Q. Gong, H. Li, H. Chen, Y. Teng, and N. Wang, “Application of Ritz method for vibration analysis of stepped functionally graded spherical torus shell with general boundary conditions," Composite Structures, vol. 243, p. 112215, 2020.

[36] H. Li, F. Pang, H. Chen, and Y. Du, "Vibration analysis of functionally graded porous cylindrical shell with arbitrary boundary restraints by using a semi analytical method," Composites Part B: Engineering, vol. 164, pp. 249-264, 2019.

[37] Y. Qu, X. Long, G. Yuan, and G. Meng, “A unified formulation for vibration analysis of functionally graded shells of revolution with arbitrary boundary conditions," Composites Part B: Engineering, vol. 50, pp. 381-402, 2013.

[38] Y. G. Qu et al., "A domain decomposition method for vibration analysis of conical shells with uniform and stepped thickness," Journal of Vibration and Acoustics-Transactions of the ASME, vol. 135, no. 1, 2013.

[39] H. Li, F. Pang, X. Miao, and Y. Li, "Jacobi-Ritz method for free vibration analysis of uniform and stepped circular cylindrical shells with arbitrary boundary conditions: a unified formulation," Computers \& Mathematics with Applications, vol. 77, no. 2, pp. 427-440, 2019.

[40] Y. Qu, Y. Chen, X. Long, H. Hua, and G. Meng, "Free and forced vibration analysis of uniform and stepped circular cylindrical shells using a domain decomposition method," Applied Acoustics, vol. 74, no. 3, pp. 425-439, 2013.

[41] F. Pang, H. Li, H. Chen, and Y. Shan, "Free vibration analysis of combined composite laminated cylindrical and spherical shells with arbitrary boundary conditions," Mechanics of Advanced Materials and Structures, pp. 1-18, 2019.

[42] S. Hosseini-Hashemi, F. Sharifpour, and M. R. Ilkhani, "On the free vibrations of size-dependent closed micro/nanospherical shell based on the modified couple stress theory," International Journal of Mechanical Sciences, vol. 115-116, pp. 501-515, 2016.
[43] H. Li, F. Pang, X. Miao, S. Gao, and F. Liu, "A semi analytical method for free vibration analysis of composite laminated cylindrical and spherical shells with complex boundary conditions," Thin-Walled Structures, vol. 136, pp. 200-220, 2019.

[44] F. Tornabene, "Free vibration analysis of functionally graded conical, cylindrical shell and annular plate structures with a four-parameter power-law distribution," Computer Methods in Applied Mechanics and Engineering, vol. 198, no. 37-40, pp. 2911-2935, 2009.

[45] H. Li, F. Pang, C. Gao, and R. Huo, "A Jacobi-Ritz method for dynamic analysis of laminated composite shallow shells with general elastic restraints," Composite Structures, vol. 242, Article ID 112091, 2020.

[46] H. Li, F. Pang, Q. Gong, and Y. Teng, "Free vibration analysis of axisymmetric functionally graded doubly-curved shells with un-uniform thickness distribution based on Ritz method," Composite Structures, vol. 225, Article ID 111145, 2019. 